\title{
Neutralinos betray their singlino nature at the ILC
}

\author{
Gudrid Moortgat-Pick, ${ }^{a, b}$ Stefano Porto ${ }^{a}$ and Krzysztof Rolbiecki ${ }^{c}$ \\ ${ }^{a}$ II. Institut für Theoretische Physik, Universität Hamburg, \\ Luruper Chaussee 149, 22761 Hamburg, Germany \\ ${ }^{b}$ DESY Hamburg, \\ Notkestraße 85, 22607 Hamburg, Germany \\ ${ }^{c}$ Instituto de Física Teórica IFT-UAM/CSIC, Universidad Autónoma de Madrid, \\ Calle Nicolás Cabrera 13-15, Cantoblanco, 28049 Madrid, Spain \\ E-mail: gudrid.moortgat-pick@desy.de, stefano.porto@desy.de, \\ krzysztof.rolbiecki@desy.de
}

ABSTRACT: It is one of the most challenging tasks at the Large Hadron Collider and at a future Linear Collider not only to observe physics beyond the Standard Model, but to clearly identify the underlying new physics model. In this paper we concentrate on the distinction between two different supersymmetric models, the MSSM and the NMSSM, as they can lead to similar low energy spectra. The NMSSM adds a singlet superfield to the MSSM particle spectrum and simplifies embedding a SM-like Higgs candidate with the measured mass of about $125.5 \mathrm{GeV}$. In parts of the parameter space the Higgs sector itself does not provide sufficient indications for the underlying model. We show that exploring the gaugino/higgsino sectors could provide a meaningful way to distinguish the two models. Assuming that only the lightest chargino and neutralino masses and polarized cross sections $e^{+} e^{-} \rightarrow \tilde{\chi}_{i}^{0} \tilde{\chi}_{j}^{0}, \tilde{\chi}_{i}^{+} \tilde{\chi}_{j}^{-}$are accessible at the linear collider, we reconstruct the fundamental MSSM parameters $M_{1}, M_{2}, \mu, \tan \beta$ and study whether a unique model distinction is possible based on this restricted information. Depending on the singlino admixture in the lightest neutralino states, as well as their higgsino or gaugino nature, we define several classes of scenarios and study the prospects of experimental differentiation.

KeYwords: Supersymmetry Phenomenology

ArXiv EPrint: 1406.7701 


\section{Contents}

1 Introduction 1

2 Strategy 2

3 Classes of scenarios 5

3.1 Light singlino scenario 6

3.2 Light higgsino scenario, $\mu_{\text {eff }}<M_{1}<M_{2} \quad 8$

3.3 Light gaugino scenario, $\mu_{\mathrm{eff}}>M_{1}>M_{2} \quad 14$

4 Conclusions and outlook $\quad 17$

A Chargino, neutralino and Higgs sector 18

$\begin{array}{ll}\text { A.1 Chargino and neutralino mass matrices } & 18\end{array}$

A.2 $\mathbb{Z}_{\mathbf{3}}$-NMSSM Higgs sector 19

$\begin{array}{lr}\text { B Scenarios } & 20\end{array}$

$\begin{array}{ll}\text { B.1 Light singlino scenario } & 20\end{array}$

$\begin{array}{ll}\text { B.2 Light higgsino scenario } & 20\end{array}$

$\begin{array}{lll}\text { B.3 Light gaugino scenario } & 21\end{array}$

\section{Introduction}

The discovery of a neutral scalar particle $[1,2]$ with mass $\sim 125.5 \mathrm{GeV}$ at the Large Hadron Collider (LHC) [3, 4] has opened a plethora of discussions about its identity. While the experimental uncertainty suggests the new particle to be the Standard Model Higgs boson, more data are still needed to precisely determine its branching ratios, the CP properties and the underlying model. The present results are in fact compatible with one of the most promising Beyond the Standard Model candidates: supersymmetry (SUSY) [5]. The latter solves - contrary to the Standard Model (SM) - the electroweak hierarchy puzzle, offers a dark matter candidate and is consistent with grand unification of the gauge couplings.

The most studied supersymmetric models are the Minimal Supersymmetric Standard Model (MSSM) [5] and its minimal extension, the Next-to-Minimal Supersymmetric Standard Model (NMSSM) [6]. The NMSSM introduces a gauge singlet chiral supermultiplet $\tilde{S}$ that allows for a relaxation of the electroweak fine tuning conditions, compared to the MSSM. On the other hand, the - so far - negative result of LHC searches for physics beyond the Standard Model (BSM) [7, 8] does not favor any of these models a priori.

In case of SUSY discovery at the LHC and/or at a linear collider (LC) it is therefore important to understand how to entail the underlying supersymmetric model, in particular 
how to distinguish between NMSSM and MSSM. These two models have indeed a very similar particle spectrum, with the exception for the superfield $\hat{S}$ in the NMSSM that results in three additional states with respect to the MSSM: a CP-even Higgs, a CP-odd Higgs and a fifth neutralino.

It is therefore well-motivated to look at the Higgs sector, where the experiments are expected to give the most precise indications $[9,10]$, and to complement the information by studying the (extended) neutralino sector of the NMSSM to look for deviations with respect to the MSSM.

Concerning the gaugino/higgsino sector, it has been shown that detecting the lightest chargino $\tilde{\chi}_{1}^{ \pm}[11]$, and neutralino states $\tilde{\chi}_{1}^{0}, \tilde{\chi}_{2}^{0}[12,13]$, a full reconstruction of the MSSM chargino and neutralino sectors through $\chi^{2}$-fits $[14,15]$ is possible based on measuring the masses and their polarized cross sections. A fit disfavouring the MSSM suggests to look at minimal extensions that modify the neutralino/chargino sector, in primis the NMSSM [16, 17].

In fact, the singlino admixtures of neutralino lightest states as well as the higgsino and gaugino components of $\tilde{\chi}_{1}^{0}$ allow to identify several classes of NMSSM scenarios. Scenarios where the singlino component in the light neutralinos is significant (light singlino scenarios), are often treated in the literature, featuring production cross sections and phenomenology different from the MSSM and are in principle easier to spot. If the singlino, however, is heavy and mainly present in $\tilde{\chi}_{3}^{0}, \tilde{\chi}_{4}^{0}$ or $\tilde{\chi}_{5}^{0}$, the phenomenology is more MSSM-like and we distinguish the cases where the main component of the lightest $\tilde{\chi}_{1}^{0}$ is higgsino-like (light higgsino scenarios) or gaugino-like (light gaugino scenarios). Having a decoupled singlino may result in a scenario that is experimentally not distinguishable from the MSSM without further information about the heavier neutralino states and the Higgs sector. Our analysis confirms these hints, concluding that a light and accessible singlino is one of the most efficient ways for model distinction together with a light singlet scalar.

The paper is organized in the following way: first we introduce our proposed strategy to discriminate the different models in section 2 and describe the classes of scenarios in section 3. In that section we also try to clearly classify in which cases a unique distinction between both models is possible based only on the light electroweak states and to work out which further information is required in cases where the light sector alone does not provide sufficient information for a model discrimination. Therefore we perform scans in the $(\lambda, \kappa)$-plane, applying the most recent phenomenological and experimental constraints from colliders, including also dark matter experiments, and determine where the singlino admixtures are such that the NMSSM cannot be misinterpreted as MSSM. We summarize our results in section 4 and list details and parameters on the models in the appendices A, B.

\section{$2 \quad$ Strategy}

As explained in the Introduction, the NMSSM adds to the MSSM an additional gauge singlet superfield $\hat{S}$ in the Higgs sector. The most studied version of the NMSSM has a Lagrangian with an accidental $\mathbb{Z}_{3}$ symmetry, obtained from the scale invariant superpo- 
tential [6],

$$
W_{\mathbb{Z}_{3-\mathrm{NMSSM}}} \supset \lambda \hat{S} \hat{H}_{u} \cdot \hat{H}_{d}+\frac{\kappa}{3} \hat{S}^{3} .
$$

$\hat{S}$ consists of a scalar Higgs singlet $S$ and the singlino $\tilde{S}$. The additional dimensional parameters $A_{\lambda}$ and $A_{\kappa}$ appear in the Higgs sector soft terms:

$$
\mathcal{L}_{\text {soft, } \mathbb{Z}_{3} \text {-NMSSM }} \supset-\lambda A_{\lambda} H_{u} \cdot H_{d} S-\frac{1}{3} A_{\kappa} S^{3} .
$$

The singlet $S$, see eqs. (2.1) and (2.2), mixes due to the electroweak symmetry breaking with the MSSM Higgs doublets $H_{u}, H_{d}$, resulting in three CP-even neutral scalars $h_{1}, h_{2}, h_{3}$ and two CP-odd neutral scalars $a_{1}, a_{2}$. Correspondingly, the singlino $\tilde{S}$ mixes with the higgsinos and the gauginos, resulting in five neutralino mass eigenstates. Therefore, determining the nature of weakly coupling scalars or neutralinos is the first way to discriminate between NMSSM and MSSM.

In the light of the expected high accuracy in the Higgs sector measurements [10], it is a common practice to compare MSSM and NMSSM scenarios looking at the Higgs sector, in particular at the Higgs decays [18-22]. The case in which a very light CP-even and/or a light CP-odd scalars have high singlet component and allow new decay channels for the SMlike Higgs scalar affecting its decay width and branching ratios has been explored [23]. On the same footing, looking at the extended NMSSM neutralino sector is very well motivated, especially for linear collider phenomenology, due to the high precision in the electroweak sector. This can be crucial in case of relatively heavy singlet states in comparison with the SM-like Higgs, such that the observed Higgs sector can be interpreted within both the MSSM and the NMSSM. In such scenarios with heavy decoupled states, the corresponding signatures at the LHC would indeed be very similar in both models [10].

We are therefore interested to understand how much information can be obtained from the neutralino and chargino sector for the model distinction. In the MSSM, the parameters $M_{1}, M_{2}, \mu, \tan \beta$ fully describe the chargino and neutralino sector. One should note that these are fundamental parameters without any assumption on the SUSY breaking scheme. Precise determination of these parameters is possible at a linear collider [11-14, 24], provided that $\tilde{\chi}_{1}^{0}, \tilde{\chi}_{2}^{0}$ and $\tilde{\chi}_{1}^{ \pm}$can be produced at the LC and their masses as well as the polarized cross sections $\sigma\left(e^{+} e^{-} \rightarrow \tilde{\chi}_{1}^{0} \tilde{\chi}_{2}^{0}\right), \sigma\left(e^{+} e^{-} \rightarrow \tilde{\chi}_{1}^{+} \tilde{\chi}_{1}^{-}\right)$are measured. An accurate and rather model-independent determination of $M_{1}, M_{2}, \mu, \tan \beta$ is performed by a $\chi^{2}-$ minimisation that selects the parameters fitting the experimental results. Such analysis can be strengthened if the mass of the heavier neutralino states can be inferred from combined analyses of LHC and LC data [14].

The possibility of reconstructing the MSSM chargino-neutralino sector parameters can then be exploited as a tool for the distinction between the MSSM and the NMSSM [16]. Given experimental observation of $\tilde{\chi}_{1}^{0}, \tilde{\chi}_{2}^{0}$ and $\tilde{\chi}_{1}^{ \pm}$, a result of the $\chi^{2}$-fit that excludes the MSSM at $95 \%$ confidence level (C.L.), may suggest the NMSSM. It has indeed been shown [16] that relatively different mixing for MSSM and NMSSM scenarios can lead to very similar neutralino and chargino mass spectra in both models; this is of course also true in case of a scenarios with similar soft parameters and a decoupled singlet superfield.

Following this idea, we outline our strategy: 
- Scenario selection. We identify NMSSM scenarios that present a mass spectrum for $\tilde{\chi}_{1}^{ \pm}, \tilde{\chi}_{1}^{0}, \tilde{\chi}_{2}^{0}$ and low Higgs spectrum that can be attributed also to a MSSM scenario. We calculate the corresponding NMSSM neutralino and chargino tree-level masses and polarized cross-sections for the processes $e^{+} e^{-} \rightarrow \tilde{\chi}_{1}^{+} \tilde{\chi}_{1}^{-}$and $e^{+} e^{-} \rightarrow \tilde{\chi}_{1}^{0} \tilde{\chi}_{2}^{0} \mathrm{cf}$. (figures 1 and 2).

- Constraints. Each scenario has to fulfill a series of phenomenological and experimental constraints implemented in NMSSMTools-4.2.1, that includes NMHDECAY [25-27] and NMSDECAY $[28,29]$. These tools calculate the Higgs sector parameters, SUSY particle masses at the loop level and their decays, and confront them with limits from LEP, LHC and EW precision constraints. An interface to MicrOMEGAS [30] provides dark matter constraints, including the latest LUX [31] and Planck [32] results. The LSP relic density is required to be $\Omega_{\mathrm{LSP}} h^{2}<0.131$, where $h$ is the Hubble constant in units of $100 \mathrm{~km} /(\mathrm{s} \cdot \mathrm{Mpc})$. Higgs sector constraints are further controlled using HiggsBounds-4.0.0 [33] and HiggsSignals-1.0.0 [34], such that a scenario is accepted only if compatible with current data at the $95 \%$ (C.L.).

- Experimental assumption. We assume, for each NMSSM scenario, an observation of $\tilde{\chi}_{1}^{ \pm}, \tilde{\chi}_{1}^{0}$ and $\tilde{\chi}_{2}^{0}$ at the ILC together with their total cross sections $\sigma\left(e^{+} e^{-} \rightarrow\right.$ $\left.\tilde{\chi}_{1}^{+} \tilde{\chi}_{1}^{-}\right), \sigma\left(e^{+} e^{-} \rightarrow \tilde{\chi}_{1}^{0} \tilde{\chi}_{2}^{0}\right)$ with electron-positron beam polarizations $\left(\mathcal{P}_{e^{-}}, \mathcal{P}_{e^{+}}\right)=$ $( \pm 0.9, \mp 0.55)$ at $\sqrt{s}=350 \mathrm{GeV}$ (corresponding to the $t \bar{t}$-threshold) and at $\sqrt{s}=$ $500 \mathrm{GeV}$. A precision of $0.5 \%$ on the masses and $1 \%$ on the cross sections is assumed $[35,36]$. If kinematically accessible, also $m_{\tilde{\chi}_{3}^{0}}$, and the processes $e^{+} e^{-} \rightarrow \tilde{\chi}_{1}^{0} \tilde{\chi}_{3}^{0}$, $e^{+} e^{-} \rightarrow \tilde{\chi}_{2}^{0} \tilde{\chi}_{3}^{0}$ are considered.

- $\chi^{2}$-fit to MSSM. The measured quantities and errors are used to perform a MSSM parameter determination through the $\chi^{2}$-fit following the recipe in [14], similarly to [16]. We apply the $\chi^{2}$-fit using Minuit [37], that minimizes the $\chi^{2}$ function defined as

$$
\chi^{2}=\sum_{i}\left|\frac{\mathcal{O}_{i}-\overline{\mathcal{O}}_{i}}{\delta \mathcal{O}_{i}}\right|^{2},
$$

where $\mathcal{O}_{i}$ are the input observables, $\delta O_{i}$ are the associated experimental uncertainties and $\bar{O}_{i}$ are the theoretical values of the observables calculated using the fitted MSSM parameters. The unknowns of the fit will be $M_{1}, M_{2}, \mu, \tan \beta$ and $m_{\tilde{\nu}_{e}}{ }^{1}$ In the case of high $\tan \beta$, its extraction could be difficult, and only a lower limit could be set. A fit that is not consistent with the MSSM 95\% C.L., may give hints towards the NMSSM and model distinction. If this is not the case, more information is needed to be included to establish the nature of the observed model. The limiting $(95 \%$ C.L.) value of $\chi^{2}$ varies for different scenarios under consideration depending on the number of observables used in the fit.

- Information from the Higgs sector. If the singlet is relatively light and has a substantial mixing with the SM-like Higgs, one could observe deviations from the SM

\footnotetext{
${ }^{1}$ The mass $m_{\tilde{\nu}_{e}}$ is related to the selectron masses by applying the $\mathrm{SU}(2)$ relation $m_{\tilde{\nu}_{e}}^{2}=m_{\tilde{e}_{L}}^{2}+$ $\cos (2 \beta) \cos ^{2} \theta_{W} m_{Z}^{2}$ and $m_{\tilde{e}_{L}}=m_{\tilde{e}_{R}}$.
} 

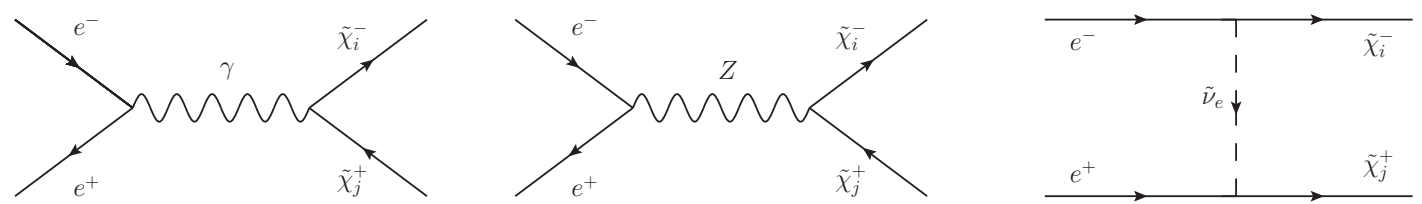

Figure 1. Chargino tree-level production channels at $e^{+} e^{-}$colliders.
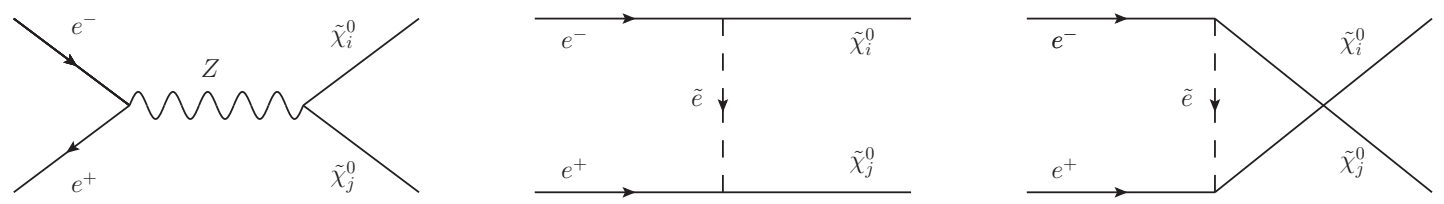

Figure 2. Neutralino tree-level production channels at $e^{+} e^{-}$colliders.

predictions that cannot be accommodated within the MSSM at the same time. In our case, we expect small departure from the SM values and we limit ourselves to comparing the NMSSM predictions to the SM model by doing a $\chi^{2}$-fit of the reduced couplings of the SM-like Higgs to $g, \gamma, W, Z, b, c, \tau$. If the couplings do not differ too much from the SM, such a scenario could always be accommodated within the MSSM in the decoupling limit as well. Alternatively, one could consider a possibility of detection additional singlet-like states, but this analysis is beyond the scope of the current paper.

\section{Classes of scenarios}

The singlino $(\tilde{S})$ admixtures of the lightest neutralino states $\tilde{\chi}_{1}^{0}$ and $\tilde{\chi}_{2}^{0}$ suggest the classification of NMSSM scenarios with the following limiting cases:

1. Light singlino $(L S)$ scenarios: high $\tilde{S}$ admixture in the light states $\tilde{\chi}_{1}^{0}$ or $\tilde{\chi}_{2}^{0}$.

2. Light higgsino (LH) scenarios: higgsino-like $\tilde{\chi}_{1}^{0}$, with $\mu_{\mathrm{eff}}<M_{1}, M_{2}$ and high $\tilde{S}$ admixture mainly in $\tilde{\chi}_{3}^{0}, \tilde{\chi}_{4}^{0}, \tilde{\chi}_{5}^{0}$.

3. Light gaugino (LG) scenarios: gaugino-like $\tilde{\chi}_{1}^{0}$, with $\mu_{\mathrm{eff}}>M_{1}, M_{2}$ and high $\tilde{S}$ admixture mainly in $\tilde{\chi}_{3}^{0}, \tilde{\chi}_{4}^{0}, \tilde{\chi}_{5}^{0}$.

Exploring these classes of scenarios allows to embed also the intermediate cases of mixed lightest neutralino nature.

A high singlino admixture in $\tilde{\chi}_{1}^{0}$ and/or $\tilde{\chi}_{2}^{0}$ as in case 1 may signal beyond-MSSM physics. A fit reconstructing the higgsino and gaugino components hypothesizing MSSM would give very different result with respect to the original NMSSM. In such a case, the outlined strategy for model distinction seems promising, see [16] and section 3.1, as different gaugino and neutralino admixtures lead to modified cross sections, production channels, as well as decays. 


\begin{tabular}{|c|c|c|c|c|c|c|}
\hline & $M_{1}[\mathrm{GeV}]$ & $M_{2}[\mathrm{GeV}]$ & $\mu, \mu_{\mathrm{eff}}=\lambda \cdot x[\mathrm{GeV}]$ & $\tan \beta$ & $\lambda$ & $\kappa$ \\
\hline MSSM & 406 & 115.8 & 354 & 8 & - & - \\
\hline NMSSM & 365 & 111 & 484 & 9.5 & 0.16 & 0.0585 \\
\hline
\end{tabular}

Table 1. Neutralino and chargino parameters for the NMSSM scenario LS and for the corresponding MSSM scenario.

In cases 2 and 3 , instead, both spectra and admixtures of the detected states $\tilde{\chi}_{1}^{0}$ and $\tilde{\chi}_{2}^{0}$ could result in a MSSM-like phenomenology, therefore it is likely that the fit is still compatible with the MSSM, see subsections 3.2 and 3.3. In these cases one should ask how to efficiently integrate informations from heavier neutralino states, and/or from the Higgs sector.

Given a fixed $\mu_{\text {eff }}=\lambda s$, the key parameters of the NMSSM neutralino sector are $\lambda$ and $\kappa$ as they regulate the singlino admixture in the mass eigenstates, see the NMSSM neutralino mass matrix, eq. (A.7) in appendix A. In two heavy-singlino cases, see examples in subsections 3.2 and 3.3 , we scan a grid of ten thousand points in the $(\lambda, \kappa)$-plane for values $\lambda \in[0,0.7]$ and $\kappa \in[0,0.7]$, to study how the model discrimination method works at the ILC along the $(\lambda, \kappa)$-plane, as the singlino admixtures vary. For each point passing the previous phenomenological and experimental constraints, we perform the $\chi^{2}$-fit described above. These scans allow to see how the singlino "mass" vary along the $(\lambda, \kappa)$-plane, and to observe areas in which the singlino is mostly very heavy and decoupled, areas in which the singlino is placed among the lightest neutralino states, as well as regions with mixed behaviour.

\subsection{Light singlino scenario}

As a first example, we choose an NMSSM scenario with wino $\tilde{\chi}_{1}^{0}$ but with high singlino components in $\tilde{\chi}_{2}^{0}$ (and $\tilde{\chi}_{3}^{0}$ ). We refer to it as the light singlino scenario (LS). The lower neutralino/chargino spectrum can be reproduced by an MSSM scenario with different $M_{1}, M_{2}, \mu, \tan \beta$, see table 1 . Both for LS and the corresponding MSSM scenario we have $M_{1}>M_{2}$, as it is common in AMSB models. We set $A_{\lambda}=4200 \mathrm{GeV}$ and $A_{\kappa}=-200 \mathrm{GeV}$. For the remaining parameters of the NMSSM scenario, we refer to appendix B.1. A SM-like Higgs with $m_{h}=125 \mathrm{GeV}$ is reproduced.

The Higgs spectrum is given in table $2 .^{2}$ The mass $m_{h_{1}}$ can be easily reproduced within the corresponding MSSM scenario with a proper choice of the stop soft parameters. The states $h_{2}$ and $a_{1}$, being both $\sim 100 \%$ singlets, are not expected to be visible both at the LHC and ILC because they are not directly coupling to other particles and are relatively heavy. A detailed analysis could point a way to observe these states but this is beyond scope of this work.

The tree-level masses for the neutralino/chargino sector are listed in table 3 . The light part of the spectrum is nearly indistinguishable between the two models, with $\tilde{\chi}_{1}^{0} \sim \tilde{W}$.

\footnotetext{
${ }^{2}$ In this study we used: $m_{t}=173.07 \mathrm{GeV}, \quad m_{Z}=91.1876 \mathrm{GeV}, \quad \Gamma_{Z}=2.4952 \mathrm{GeV}, \quad m_{W}=80.385$, $\Gamma_{W}=2.085 \mathrm{GeV}, \alpha_{\mathrm{em}}=1 / 127.92, \alpha_{\mathrm{S}}\left(m_{Z}\right)=0.1184$, with $\sin ^{2} \theta_{W}=1-m_{W}^{2} / m_{Z}^{2}$.
} 


\begin{tabular}{|l|c|c|c|c|c|c|}
\hline & $m_{h_{1}}[\mathrm{GeV}]$ & $m_{h_{2}}[\mathrm{GeV}]$ & $m_{h_{3}}[\mathrm{GeV}]$ & $m_{a_{1}}[\mathrm{GeV}]$ & $m_{a_{2}}[\mathrm{GeV}]$ & $m_{H^{ \pm}}[\mathrm{GeV}]$ \\
\hline NMSSM & 124.9 & 303.0 & 4467.3 & 324.0 & 4467.3 & 4468.1 \\
\hline
\end{tabular}

Table 2. LS scenario: Higgs spectrum calculated at the 1-loop level with full 2-loops contributions from bottom/top Yukawa couplings with NMSSMTools [25-27].

\begin{tabular}{|c|c|c|c|c|c|c|c|}
\hline & $m_{\tilde{\chi}_{1}^{0}}[\mathrm{GeV}]$ & $m_{\tilde{\chi}_{2}^{0}}[\mathrm{GeV}]$ & $m_{\tilde{\chi}_{3}^{0}}[\mathrm{GeV}]$ & $m_{\tilde{\chi}_{4}^{0}}[\mathrm{GeV}]$ & $m_{\tilde{\chi}_{5}^{0}}[\mathrm{GeV}]$ & $m_{\tilde{\chi}_{1}^{ \pm}}[\mathrm{GeV}]$ & $m_{\tilde{\chi}_{2}^{ \pm}}[\mathrm{GeV}]$ \\
\hline MSSM & 104.8 & 350.4 & 360.1 & 426.7 & - & 105.1 & 375.0 \\
\hline NMSSM & 104.9 & 350.1 & 360.5 & 489.7 & 504.1 & 105.1 & 498.5 \\
\hline
\end{tabular}

Table 3. Neutralino and chargino masses in the LS scenario and in the corresponding reference MSSM scenario. The mass difference $m_{\tilde{\chi}_{1}^{ \pm}}-m_{\tilde{\chi}_{1}^{0}}$ receives significant positive NLO corrections. Here, we only use tree-level masses, however for such a quasi-degenerate states the mass measurement usually has a larger uncertainty than the mass difference itself so in a more realistic setting one should use the mass difference as an input rather than the actual masses, see e.g. ref. [38].

\begin{tabular}{|c|c|c|}
\hline & MSSM & NMSSM \\
\hline$\tilde{\chi}_{1}^{0}$ & $\sim 93 \% \tilde{W}$ & $\sim 97 \% \tilde{W}$ \\
\hline$\tilde{\chi}_{2}^{0}$ & $\sim 26 \% \tilde{B}+69 \% \tilde{H}_{u, d}$ & $\sim 22 \% \tilde{B}+73 \% \tilde{S}$ \\
\hline$\tilde{\chi}_{3}^{0}$ & $\sim \tilde{H}_{u, d}$ & $\sim 72 \% \tilde{B}+25 \% \tilde{S}$ \\
\hline
\end{tabular}

Table 4. The dominant admixtures of the three lightest neutralinos in the LS scenario and in the corresponding MSSM scenario.

However, the other lighter states $\tilde{\chi}_{2}^{0}, \tilde{\chi}_{3}^{0}$ feature different admixtures, see table 4 , leading to different production cross sections and relative importance of the production channels.

We take $m_{\tilde{e}_{L}}=303.5 \mathrm{GeV}$, assuming $m_{\tilde{e}_{L}}=m_{\tilde{e}_{R}}$ and $m_{\tilde{\nu}_{e}}^{2}=m_{\tilde{e}_{L}}^{2}+\cos (2 \beta) \cos ^{2} \theta_{W} m_{Z}^{2}$. The production cross sections are listed in table 5 . For the fit to the MSSM we only include NMSSM cross sections larger than $1 \mathrm{fb}$. The relatively light NMSSM $\tilde{\chi}_{3}^{0}$ can be produced with a sizeable cross section at $500 \mathrm{GeV}$, therefore we also include $\sigma\left(e^{+} e^{-} \rightarrow \tilde{\chi}_{1}^{0} \tilde{\chi}_{3}^{0}\right)$ for $P=(-0.9,0.55)$ in the fit.

The fitted MSSM parameters are then

$$
\begin{aligned}
M_{1}=430.0 \pm 1.6 \mathrm{GeV}, & M_{2}=111.8 \pm 0.8 \mathrm{GeV} \\
\mu=370.4 \pm 0.7 \mathrm{GeV}, & m_{\nu_{e}}=310.6 \pm 2.8 \mathrm{GeV}
\end{aligned}
$$

and $\tan \beta$ remains unconstrained. These parameters would be consistent with neutralino and chargino masses in the MSSM, listed in table 6.

The fit with $10-5=5$ degrees of freedom (d.o.f.) gives $\chi^{2}=62.6$, clearly stating that the hypothesized model (MSSM) is not compatible with the experimental data (with the $95 \%$ C.L. being $\chi^{2}<11.1$ ). This could be additionally confirmed by the mass of the heavy neutralino, $m_{\tilde{\chi}_{4}^{0}}$, if it is eventually measured at the higher center-of-mass energy. Additionally, we note that the predicted mass of the heavy chargino, $m_{\tilde{\chi}_{2}^{ \pm}}=389.1 \mathrm{GeV}$, makes production of the mixed chargino pair, $\tilde{\chi}_{1}^{ \pm} \tilde{\chi}_{2}^{\mp}$ possible. The expected cross section, $\sim 3 \mathrm{fb}$, could in principle allow for its measurement at $\sqrt{s}=500 \mathrm{GeV}$. The non-observation 


\begin{tabular}{|c|c|c|c|c|c|}
\hline \multicolumn{7}{|c|}{$\sigma\left(e^{+} e^{-} \rightarrow \tilde{\chi}_{1}^{+} \tilde{\chi}_{1}^{-}\right)$} & {$[\mathrm{fb}]$} \\
\hline$\sqrt{s}=350 \mathrm{GeV}$ & MSSM & NMSSM & $\sqrt{s}=500 \mathrm{GeV}$ & MSSM & NMSSM \\
\hline$P=(-0.9,0.55)$ & 2491.0 & 2575.3 & $P=(-0.9,0.55)$ & 1165.4 & 1213.0 \\
\hline$P=(0.9,-0.55)$ & 39.5 & 42.4 & $P=(0.9,-0.55)$ & 18.3 & 18.8 \\
\hline
\end{tabular}

\begin{tabular}{|c|c|c|c|c|c|}
\hline \multicolumn{3}{|c|}{$\sigma\left(e^{+} e^{-} \rightarrow \tilde{\chi}_{1}^{0} \tilde{\chi}_{2}^{0}\right) \quad[\mathrm{fb}]$} & \multicolumn{3}{|c|}{$\sigma\left(e^{+} e^{-} \rightarrow \tilde{\chi}_{1}^{0} \tilde{\chi}_{3}^{0}\right) \quad[\mathrm{fb}]$} \\
\hline$\sqrt{s}=500 \mathrm{GeV}$ & MSSM & NMSSM & $\sqrt{s}=500 \mathrm{GeV}$ & MSSM & NMSSM \\
\hline$P=(-0.9,0.55)$ & 24.1 & 8.6 & $P=(-0.9,0.55)$ & 25.1 & 15.0 \\
\hline$P=(0.9,-0.55)$ & 0.4 & 0.1 & $P=(0.9,-0.55)$ & 5.7 & 0.2 \\
\hline
\end{tabular}

Table 5. The production cross sections of $e^{+} e^{-} \rightarrow \tilde{\chi}_{1}^{+} \tilde{\chi}_{1}^{-}, \tilde{\chi}_{1}^{0} \tilde{\chi}_{2}^{0}, \tilde{\chi}_{1}^{0} \tilde{\chi}_{3}^{0}$ in the LS scenario and the corresponding MSSM scenario at $\sqrt{s}=350$ and $500 \mathrm{GeV}$.

\begin{tabular}{|l|c|c|c|c|c|c|}
\hline & $m_{\tilde{\chi}_{1}^{0}}[\mathrm{GeV}]$ & $m_{\tilde{\chi}_{2}^{0}}[\mathrm{GeV}]$ & $m_{\tilde{\chi}_{3}^{0}}[\mathrm{GeV}]$ & $m_{\tilde{\chi}_{4}^{0}}[\mathrm{GeV}]$ & $m_{\tilde{\chi}_{1}^{ \pm}}[\mathrm{GeV}]$ & $m_{\tilde{\chi}_{2}^{ \pm}}[\mathrm{GeV}]$ \\
\hline MSSM $_{\text {fit }}$ & 106.0 & 368.0 & 378.0 & 445.9 & 106.1 & 389.1 \\
\hline
\end{tabular}

Table 6. MSSM neutralino and chargino masses based on the resulting parameters from the fit, see eq. (3.1).

would provide another hint of the non-minimal nature of chargino/neutralino sector. A nonminimal nature of the neutralino sector would be required to explain the measurements with one of the possible candidates being NMSSM. This first example shows that an effective model distinction in the case of high admixture of singlino in the lightest neutralino is possible exploiting the outlined procedure.

\subsection{Light higgsino scenario, $\mu_{\mathrm{eff}}<M_{1}<M_{2}$}

We consider here an NMSSM scenario with a light higgsino (LH), whose chargino/neutralino parameters are:

$$
M_{1}=450 \mathrm{GeV}, \quad M_{2}=1600 \mathrm{GeV}, \quad \mu_{\mathrm{eff}}=\lambda s=120 \mathrm{GeV}, \quad \tan \beta=27,
$$

while we have $\lambda \in[0,0.7]$ and $\kappa \in[0,0.7]$ as described above; $\mu_{\text {eff }}$ is kept fixed by varying the singlet vacuum expectation value (vev) $s$. The $\hat{S}$ soft parameters are $A_{\lambda}=3000 \mathrm{GeV}$, $A_{\kappa}=-30 \mathrm{GeV}$. The first generation sfermion masses, needed for the production cross sections, are set to

$$
m_{\tilde{e}_{L}}=303.5 \mathrm{GeV}, \quad m_{\tilde{e}_{L}}=m_{\tilde{e}_{R}}, \quad m_{\tilde{\nu}_{e}}^{2}=m_{\tilde{e}_{L}}^{2}+\cos (2 \beta) \cos ^{2} \theta_{W} m_{Z}^{2},
$$

while squarks masses are $>1 \mathrm{TeV}$. For the full set of soft parameters, we refer to appendix B.2.

In figure 3 we show the result of the scan in the $\operatorname{NMSSM}(\lambda, \kappa)$-plane after our tests. Light-blue-shaded area corresponds to points that pass DM constraints; ${ }^{3}$ the points within

\footnotetext{
${ }^{3}$ Here and in the following, we allow DM density to be below Planck [32] measured value.
} 


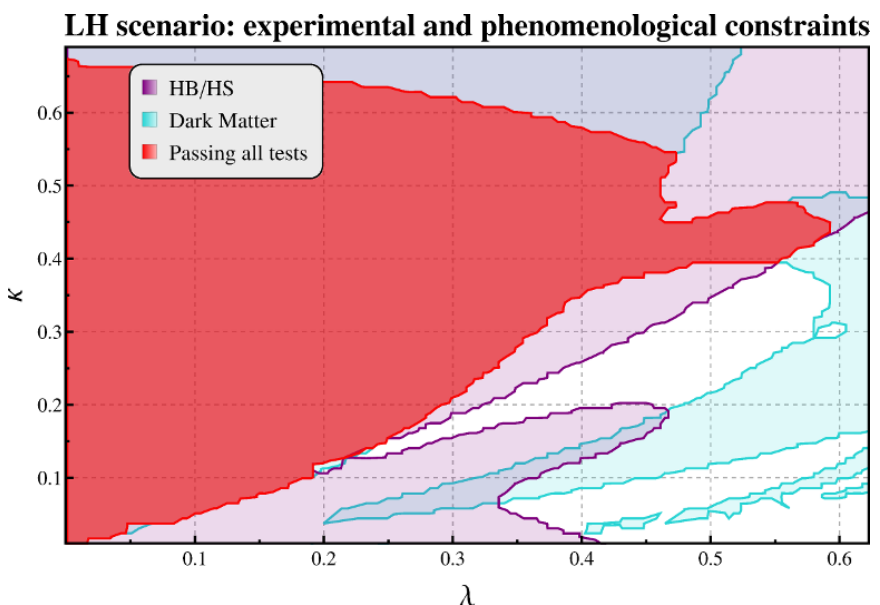

Figure 3. Light higgsino scenario: regions in the $(\lambda, \kappa)$-plane allowed by experimental and phenomenological constraints. The light-blue-shaded regions delimited by the light blue boundary pass dark matter constraints. The coloured regions delimited by the purple boundary pass checks within HiggsBounds and HiggsSignals. The red area is allowed by all the constraints.

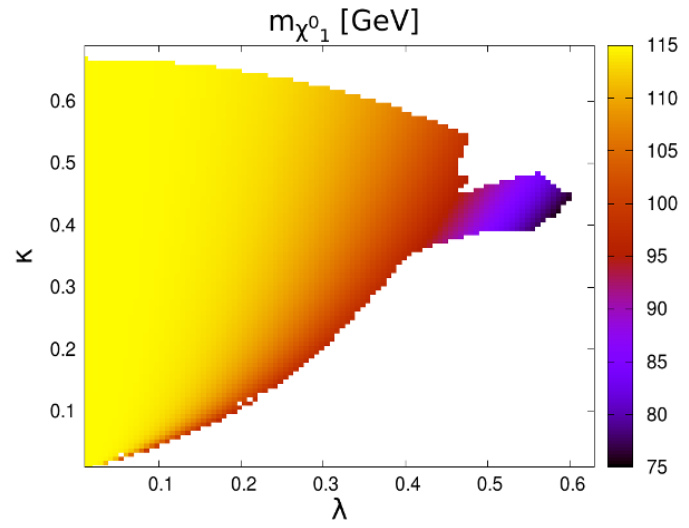

(a)

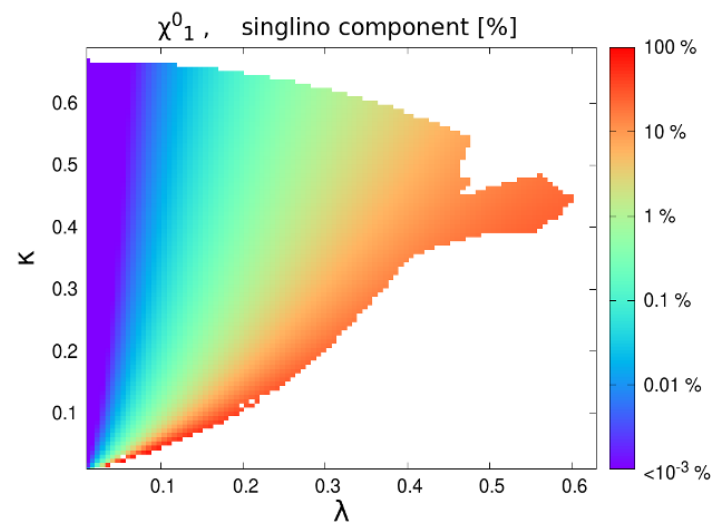

(b)

Figure 4. LH scenario: (a) the mass $m_{\tilde{\chi}_{1}^{0}}$, in $\mathrm{GeV}$; (b) the $\tilde{S}$ component of $\tilde{\chi}_{1}^{0}$, in $\%$.

purple-shaded boundary area pass the Higgs sector constraints from HiggsBounds and HiggsSignals. The solid red area is the region allowed by all the constraints, phenomenological and experimental ones, implemented within NMSSMTools, HiggsBounds and HiggsSignals.

As a reference MSSM scenario, we select the one with $M_{1}, M_{2}, \mu=\mu_{\mathrm{eff}}, \tan \beta$ and the slepton masses given in eqs. (3.2) and (3.3), to show that the light neutralino spectrum and production cross sections, see table 7 , may be very similar to the analogue quantities in the LH-NMSSM scenario in the vast part of the $(\lambda, \kappa)$-plane, (cf. figure 4 (a) for $m_{\tilde{\chi}_{1}^{0}}$ and figure 5 (a) for the corresponding cross sections).

Regarding the Higgs sector, it is possible to get a MSSM counterpart with the same SM-Higgs mass and a similar spectrum for the other Higgs states (with the exception of the new singlet states) for each point in the $(\lambda, \kappa)$-plane of the LH scenario. 


\begin{tabular}{|c|c|c|c|c|c|}
\hline$m_{\tilde{\chi}_{1}^{0}}$ & $m_{\tilde{\chi}_{2}^{0}}$ & $m_{\tilde{\chi}_{3}^{0}}$ & $m_{\tilde{\chi}_{4}^{0}}$ & $m_{\tilde{\chi}_{1}^{ \pm}}$ & $m_{\tilde{\chi}_{2}^{ \pm}}$ \\
\hline $114.8 \mathrm{GeV}$ & $123.3 \mathrm{GeV}$ & $454.4 \mathrm{GeV}$ & $1604.1 \mathrm{GeV}$ & $119.4 \mathrm{GeV}$ & $1604.1 \mathrm{GeV}$ \\
\hline
\end{tabular}

\begin{tabular}{|c|c|c|}
\hline MSSM, $\sigma\left(e^{+} e^{-} \rightarrow \tilde{\chi}_{1}^{0} \tilde{\chi}_{2}^{0}\right)$ & $\sqrt{s}=350 \mathrm{GeV}$ & $\sqrt{s}=500 \mathrm{GeV}$ \\
\hline$P=(-0.9,0.55)$ & $791.7 \mathrm{fb}$ & $391.4 \mathrm{fb}$ \\
\hline$P=(0.9,-0.55)$ & $526.7 \mathrm{fb}$ & $261.7 \mathrm{fb}$ \\
\hline
\end{tabular}

\begin{tabular}{|c|c|c|}
\hline MSSM, $\sigma\left(e^{+} e^{-} \rightarrow \tilde{\chi}_{1}^{+} \tilde{\chi}_{1}^{-}\right)$ & $\sqrt{s}=350 \mathrm{GeV}$ & $\sqrt{s}=500 \mathrm{GeV}$ \\
\hline$P=(-0.9,0.55)$ & $2348.8 \mathrm{fb}$ & $1218.9 \mathrm{fb}$ \\
\hline$P=(0.9,-0.55)$ & $445.1 \mathrm{fb}$ & $246.2 \mathrm{fb}$ \\
\hline
\end{tabular}

Table 7. The reference MSSM scenario for the LH scenario: neutralino and chargino masses [GeV] and production cross sections $\sigma\left(e^{+} e^{-} \rightarrow \tilde{\chi}_{1}^{0} \tilde{\chi}_{2}^{0}\right), \sigma\left(e^{+} e^{-} \rightarrow \tilde{\chi}_{1}^{+} \tilde{\chi}_{1}^{-}\right)[\mathrm{fb}]$.

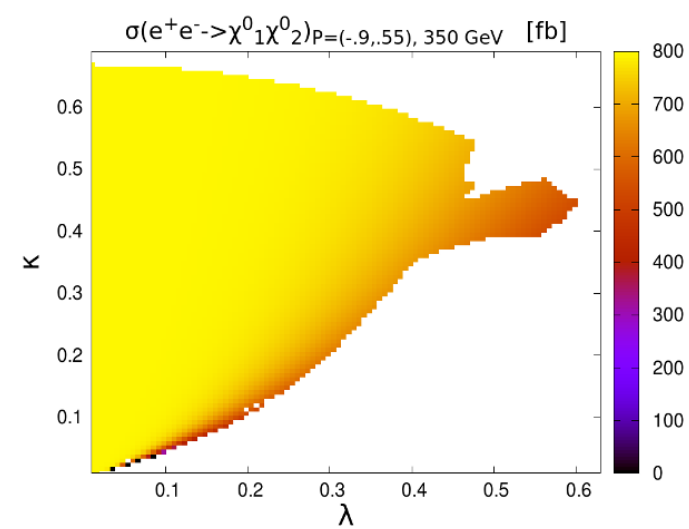

(a)

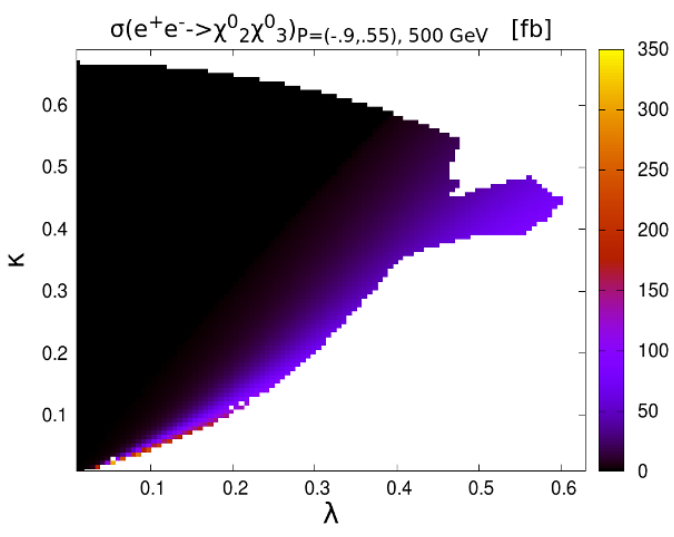

(b)

Figure 5. Production cross sections in the LH scenario: (a) $\sigma\left(e^{+} e^{-} \rightarrow \tilde{\chi}_{1}^{0} \tilde{\chi}_{2}^{0}\right)$ for $P=(-0.9,0.55)$ at $\sqrt{s}=350 \mathrm{GeV}$, in fb; (b) $\sigma\left(e^{+} e^{-} \rightarrow \tilde{\chi}_{2}^{0} \tilde{\chi}_{3}^{0}\right)$ for $P=(-0.9,+0.55)$ at $\sqrt{s}=500 \mathrm{GeV}$, in fb.

In figure 4, the NMSSM $\tilde{\chi}_{1}^{0}$ mass and its singlino component are shown. A negligible singlino component corresponds to a region in which the NMSSM $m_{\tilde{\chi}_{1}^{0}}$ is very close to the MSSM value $m_{\tilde{\chi}_{1}^{0}}=114.8 \mathrm{GeV}$. Vice versa, with a higher singlino admixture the LSP mass, $m_{\tilde{\chi}_{1}^{0}}$, within NMSSM significantly decreases.

Likewise, the neutralino polarised production cross sections $\sigma\left(e^{+} e^{-} \rightarrow \tilde{\chi}_{1}^{0} \tilde{\chi}_{2}^{0}\right)$ decrease with respect to the MSSM value following larger singlino component in $\tilde{\chi}_{1}^{0}$, see figure $5(\mathrm{a})$, as it is expected since the singlino does not couple directly to the gauge fields. The treelevel NMSSM chargino masses and production cross-sections, $\sigma\left(e^{+} e^{-} \rightarrow \tilde{\chi}_{1}^{+} \tilde{\chi}_{1}^{-}\right)$, depend only on $M_{2}, \mu_{\text {eff }}, \tan \beta$, therefore chargino production cross sections are identical to the MSSM values displayed in table 7 along all the $(\lambda, \kappa)$-plane.

According to the recipe in section 2, we assume for each point in the $(\lambda, \kappa)$-plane of the LH scenario the experimental measurement of:

- $m_{\tilde{\chi}_{1}^{0}}, m_{\tilde{\chi}_{2}^{0}}$ and $m_{\tilde{\chi}_{1}^{ \pm}}$with an uncertainty of $0.5 \%$. 


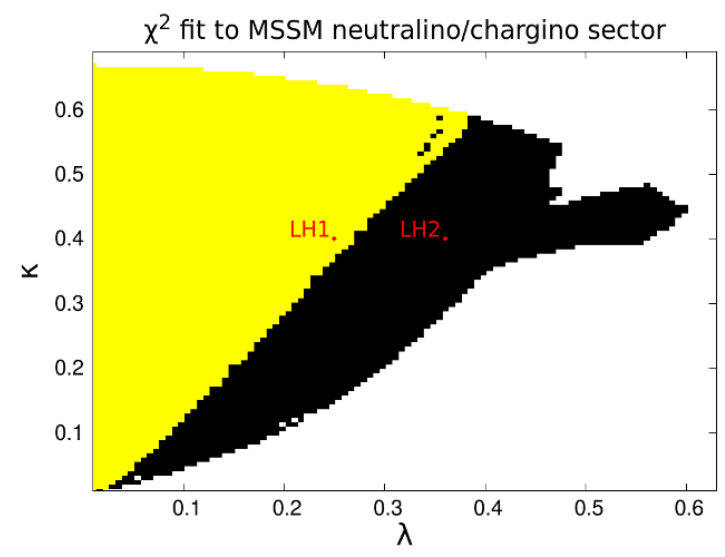

Figure 6. LH scenario: fit to the MSSM. Yellow areas are compatible with the MSSM at 95\% C.L., while black ones are excluded by the collider observables. The points LH1 $(\lambda, \kappa)=(0.25,0.4)$ and LH2 $(\lambda, \kappa)=(0.36,0.4)$ are also shown.

- $\sigma\left(e^{+} e^{-} \rightarrow \tilde{\chi}_{1}^{0} \tilde{\chi}_{2}^{0}\right)$ for $P=(\mp 0.9, \pm 0.55)$ at $\sqrt{s}=350$ and $500 \mathrm{GeV}$ with $1 \%$ uncertainty.

- $\sigma\left(e^{+} e^{-} \rightarrow \tilde{\chi}_{1}^{+} \tilde{\chi}_{1}^{-}\right)$, for $P=(\mp 0.9, \pm 0.55)$ at $\sqrt{s}=350$ and $500 \mathrm{GeV}$ with $1 \%$ uncertainty.

In the regions in which the singlino component in $\tilde{\chi}_{3}^{0}$ is higher, $m_{\tilde{\chi}_{3}^{0}}$ is decreased and $\tilde{\chi}_{2}^{0} \tilde{\chi}_{3}^{0}$ may be kinematically accessible, see figure $5(\mathrm{~b})$. In these cases, if $\tilde{\chi}_{3}^{0}$ is detectable through its decays, we consider also $m_{\tilde{\chi}_{3}^{0}}$ and $\sigma\left(e^{+} e^{-} \rightarrow \tilde{\chi}_{2}^{0} \tilde{\chi}_{3}^{0}\right)$. The production $\tilde{\chi}_{1}^{0} \tilde{\chi}_{3}^{0}$ is negligible almost everywhere. With these assumptions, a $\chi^{2}$-fit to the MSSM gives the result displayed in figure 6: the yellow areas correspond to regions in the $(\lambda, \kappa)$-plane that are at 95\% C.L. compatible with the MSSM, while in the black area MSSM is excluded. Therefore, a significant region of the parameter space, passing the implemented phenomenological and experimental constraints, can definitely be distinguished from the MSSM using collider observables. This is due to a higher singlino component in the neutralino $\tilde{\chi}_{3}^{0}$ (and partially in $\tilde{\chi}_{1}^{0}$ as well, cf. figure 4(b).

We attempt here a reconstruction of the MSSM $M_{1}, M_{2}, \mu, \tan \beta$ and $m_{\tilde{\nu}_{e}}$ for two sample points in the $(\lambda, \kappa)$-plane of the LH scenario, relatively close to the boundary between the regions of compatibility from figure 6 .

- The point LH1, with $(\lambda, \kappa)=(0.25,0.4)$, features the masses and cross sections given in tables 8 and 9. The fit to LH1 turns out to be compatible with the MSSM, $\chi^{2}=1.1$, and yields

$$
\begin{aligned}
M_{1} & =360 \pm 40 \mathrm{GeV}, & M_{2}=1300 \pm 300 \mathrm{GeV}, \\
\mu & =124 \pm 2 \mathrm{GeV}, & \tan \beta \leq 4, \\
m_{\tilde{\nu}_{e}} & \leq 470 \mathrm{GeV} . &
\end{aligned}
$$

- For our second example, the point LH2 with $(\lambda, \kappa)=(0.36,0.4)$ is taken and the corresponding masses and cross sections are given in tables 10 and 11 . The point 


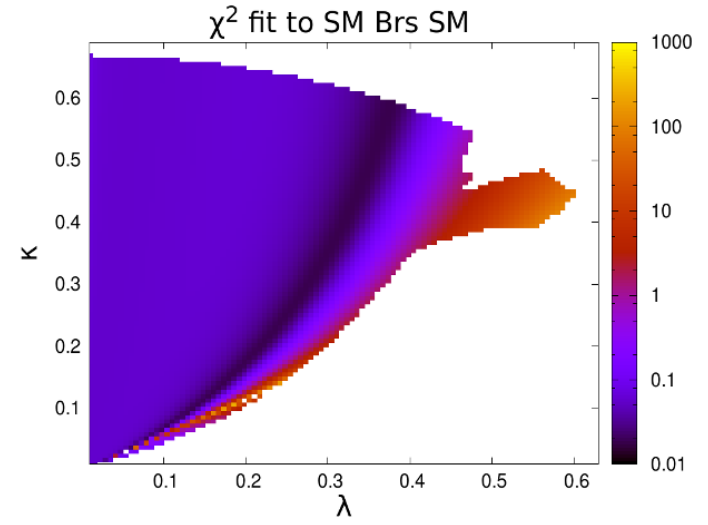

(a)

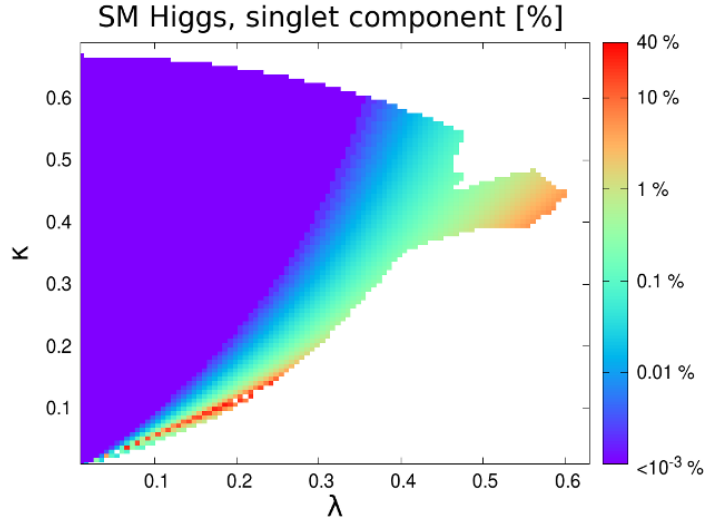

(b)

Figure 7. LH scenario: (a) 7-d.o.f. $\chi^{2}$-fit to the $\mathrm{SM}$ of the reduced couplings to $g, \gamma, W, Z, b, c, \tau$; (b) Singlet component in the SM-like Higgs, in \%.

\begin{tabular}{|c|c|c|c|c|c|c|}
\hline$m_{\tilde{\chi}_{1}^{0}}$ & $m_{\tilde{\chi}_{2}^{0}}$ & $m_{\tilde{\chi}_{3}^{0}}$ & $m_{\tilde{\chi}_{4}^{0}}$ & $m_{\tilde{\chi}_{5}^{0}}$ & $m_{\tilde{\chi}_{1}^{ \pm}}$ & $m_{\tilde{\chi}_{2}^{ \pm}}$ \\
\hline $111.6 \mathrm{GeV}$ & $125.2 \mathrm{GeV}$ & $389.0 \mathrm{GeV}$ & $454.4 \mathrm{GeV}$ & $1604 \mathrm{GeV}$ & $119.4 \mathrm{GeV}$ & $1604 \mathrm{GeV}$ \\
\hline
\end{tabular}

Table 8. Neutralino and chargino masses in the light higgsino scenario for the reference point LH1 with $(\lambda, \kappa)=(0.25,0.4)$.

\begin{tabular}{|c|c|c|}
\hline$\sigma\left(e^{+} e^{-} \rightarrow \tilde{\chi}_{1}^{0} \tilde{\chi}_{2}^{0}\right)$ & $\sqrt{s}=350 \mathrm{GeV}$ & $\sqrt{s}=500 \mathrm{GeV}$ \\
\hline$P=(-0.9,0.55)$ & $781.5 \mathrm{fb}$ & $385.8 \mathrm{fb}$ \\
\hline$P=(0.9,-0.55)$ & $519.9 \mathrm{fb}$ & $257.9 \mathrm{fb}$ \\
\hline
\end{tabular}

Table 9. Neutralino production cross sections in the light higgsino scenario, reference point LH1 with $(\lambda, \kappa)=(0.25,0.4)$.

\begin{tabular}{|c|c|c|c|c|c|c|}
\hline$m_{\tilde{\chi}_{1}^{0}}$ & $m_{\tilde{\chi}_{2}^{0}}$ & $m_{\tilde{\chi}_{3}^{0}}$ & $m_{\tilde{\chi}_{4}^{0}}$ & $m_{\tilde{\chi}_{5}^{0}}$ & $m_{\tilde{\chi}_{1}^{ \pm}}$ & $m_{\tilde{\chi}_{2}^{ \pm}}$ \\
\hline $104.2 \mathrm{GeV}$ & $128.4 \mathrm{GeV}$ & $282.4 \mathrm{GeV}$ & $454.4 \mathrm{GeV}$ & $1604 \mathrm{GeV}$ & $119.4 \mathrm{GeV}$ & $1604 \mathrm{GeV}$ \\
\hline
\end{tabular}

Table 10. Neutralino and chargino masses in the light higgsino scenario for the reference point LH2 with $(\lambda, \kappa)=(0.36,0.4)$.

LH2 in not compatible with the MSSM, with the fit giving $\chi^{2}=1700$ and the following parameter values:

$$
\begin{array}{rlrl}
M_{1} & \text { unconstrained }, & M_{2} & =317.0 \pm 0.5 \mathrm{GeV} \\
\mu & =129.3 \pm 0.6 \mathrm{GeV}, & \tan \beta<1.1 \\
m_{\tilde{\nu}_{e}} & =297 \pm 15 \mathrm{GeV} . &
\end{array}
$$

Additional information from the heavier neutralino states, such as $\tilde{\chi}_{3}^{0}$ (if its production is not already kinematically allowed at $500 \mathrm{GeV}$ ) or $\tilde{\chi}_{4}^{0}$, may help in reducing the region compatible with the MSSM. For example, given a $(\lambda, \kappa)$ coordinate and the corresponding 


\begin{tabular}{|c|c|c|}
\hline$\sigma\left(e^{+} e^{-} \rightarrow \tilde{\chi}_{1}^{0} \tilde{\chi}_{2}^{0}\right)$ & $\sqrt{s}=350 \mathrm{GeV}$ & $\sqrt{s}=500 \mathrm{GeV}$ \\
\hline$P=(-0.9,0.55)$ & $739.0 \mathrm{fb}$ & $363.3 \mathrm{fb}$ \\
\hline$P=(0.9,-0.55)$ & $491.5 \mathrm{fb}$ & $242.8 \mathrm{fb}$ \\
\hline
\end{tabular}

\begin{tabular}{|c|c|c|}
\hline$\sigma\left(e^{+} e^{-} \rightarrow \tilde{\chi}_{2}^{0} \tilde{\chi}_{3}^{0}\right)$ & $\sqrt{s}=350 \mathrm{GeV}$ & $\sqrt{s}=500 \mathrm{GeV}$ \\
\hline$P=(-0.9,0.55)$ & not accessible & $15.4 \mathrm{fb}$ \\
\hline$P=(0.9,-0.55)$ & not accessible & $10.4 \mathrm{fb}$ \\
\hline
\end{tabular}

Table 11. Neutralino production cross sections in the light higgsino scenario for the reference point LH2 with $(\lambda, \kappa)=(0.36,0.4)$.

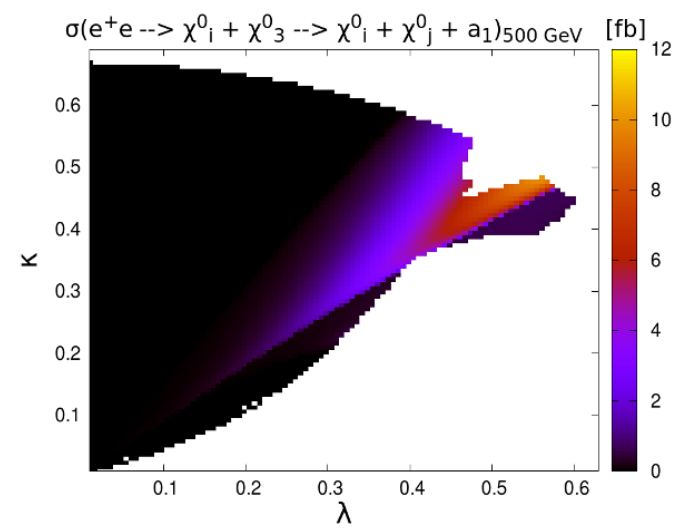

(a)

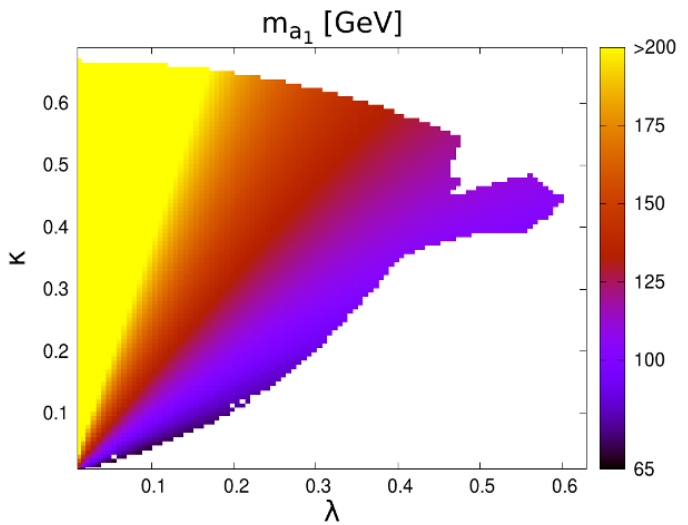

(b)

Figure 8. LH scenario: (a) inclusive cross section $e^{+} e^{-} \rightarrow \tilde{\chi}_{i}^{0} \tilde{\chi}_{3}^{0} \rightarrow \tilde{\chi}_{i}^{0} \tilde{\chi}_{j}^{0} a_{1}$ [fb], with $i, j=1,2$; (b) lightest CP-odd Higgs mass $m_{a_{1}}[\mathrm{GeV}]$.

$M_{1}, M_{2}, \mu, \tan \beta$ reconstructed from the fit, one can derive the masses of the heavier states and look for them at higher energies at the ILC or at the LHC, either confirming the fit to the MSSM or pinpointing the NMSSM. As suggested in section 2, our study may be extended by including information from the Higgs sector. A result of the naïve $\chi^{2}$-fit to the SM of the Higgs reduced couplings to $g, \gamma, W, Z, b, c, \tau$, each defined as a ratio $g_{h} / g_{h_{\mathrm{SM}}}$ between the SM-like Higgs coupling to the corresponding SM Higgs coupling, is shown in figure $7(\mathrm{a}) .{ }^{4}$ In large part of the $(\lambda, \kappa)$-plane, the SM-like Higgs of the LH scenario is compatible with the SM $\left(\chi^{2} \lesssim 14\right)$, corresponding to the MSSM-like area from the fit in figure 6. A SM-like Higgs with a higher singlet component, see figure 7(b), corresponds to a worse fit: there are two regions that are not compatible with the SM and have a different behaviour with respect of MSSM-like areas. The conclusion from this fit is therefore consistent with that of figure 6 without clearly improving our analysis.

Additional information about the NMSSM Higgs sector could obtained if new singlets are directly visible. This possibility opens up in a region with a higher singlino component in $\tilde{\chi}_{3}^{0}$, where the decays $\tilde{\chi}_{3}^{0} \rightarrow \tilde{\chi}_{1,2}^{0} a_{1}$ become open. If the production cross section for $\tilde{\chi}_{3}^{0}$ is

\footnotetext{
${ }^{4}$ We used the expected accuracies for the SM-like Higgs boson branching ratios $\Delta \mathrm{Br} / \mathrm{Br}$ from [36].
} 


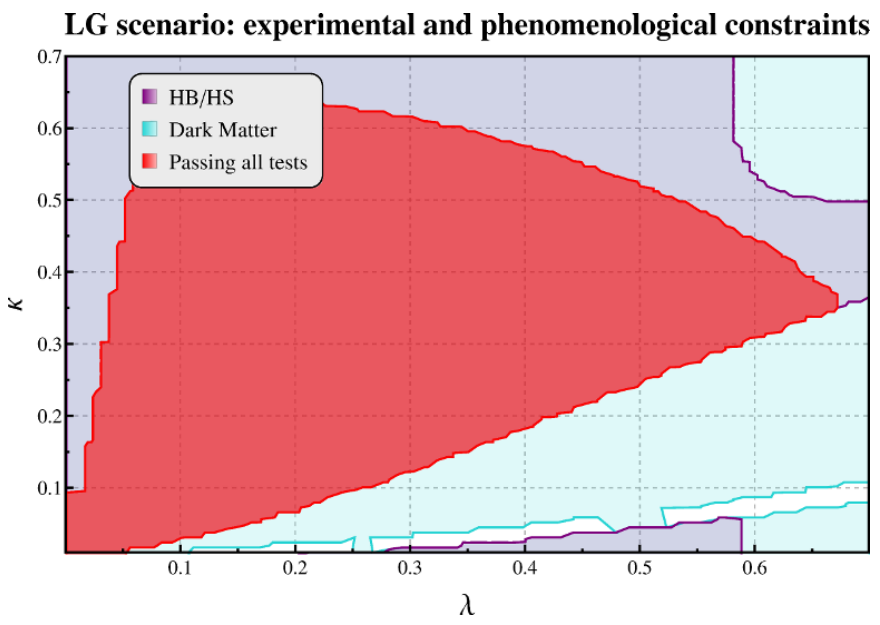

Figure 9. The light gaugino scenario: regions in the $(\lambda, \kappa)$-plane allowed by experimental and phenomenological constraints. The light-blue region passes the dark matter constraints. The purplecoloured region passes checks from HiggsBounds and HiggsSignals. The areas allowed by all the constraints are shown in red.

non-negligible one could observe the pseudoscalar $a_{1}$ via its decays $a_{1} \rightarrow b \bar{b}$. In figure 8(a) we show an inclusive cross section for production of $a_{1}$, where both production modes, $e^{+} e^{-} \rightarrow \tilde{\chi}_{1}^{0} \tilde{\chi}_{3}^{0}$ and $e^{+} e^{-} \rightarrow \tilde{\chi}_{2}^{0} \tilde{\chi}_{3}^{0}$, for both polarisations has been added up together with decays $\tilde{\chi}_{3}^{0} \rightarrow \tilde{\chi}_{1}^{0} a_{1}$ and $\tilde{\chi}_{3}^{0} \rightarrow \tilde{\chi}_{2}^{0} a_{1}$. In certain regions of parameter space, with cross sections of order $10 \mathrm{fb}$, the new state should be clearly visible. This could serve as confirmation of the NMSSM, since the MSSM would be already excluded by the fit to other observables. As a reference, in figure 8(b) we also show the mass of the pseudoscalar $a_{1}$.

\subsection{Light gaugino scenario, $\mu_{\mathrm{eff}}>M_{1}>M_{2}$}

Finally, we study an NMSSM scenario with light gauginos (LG), whose neutralino/chargino sector is given by:

$$
M_{1}=240 \mathrm{GeV}, \quad M_{2}=105 \mathrm{GeV}, \quad \mu=\mu_{\mathrm{eff}}=505 \mathrm{GeV}, \quad \tan \beta=9.2,
$$

with $\lambda \in[0,0.7]$ and $\kappa \in[0,0.7]$. The singlet soft trilinear parameters are $A_{\lambda}=3700 \mathrm{GeV}$, $A_{\kappa}=-40 \mathrm{GeV}$. The first generation sfermion masses are

$$
m_{\tilde{e}_{L}}=303.4 \mathrm{GeV}, \quad m_{\tilde{e}_{L}}=m_{\tilde{e}_{R}}, \quad m_{\tilde{\nu}_{e}}^{2}=m_{\tilde{e}_{L}}^{2}+\cos (2 \beta) \cos ^{2} \theta_{W} m_{Z}^{2},
$$

while squarks masses are $>1 \mathrm{TeV}$. For the full set of soft parameters, we refer to appendix B.3. In figure 9 we display the result of the scan in the NMSSM $(\lambda, \kappa)$-plane after our tests implemented within NMSSMTools, HiggsBounds and HiggsSignals. The colour conventions are the same as for the LH scenario, section 3.2; for the LG scenario the regions allowed by the Higgs sector constraints from HiggsBounds and HiggsSignals overlap entirely those passing DM matter constraints.

A reference MSSM scenario with an almost indistinguishable lighter (tree-level) neutralino and chargino mass spectrum and production cross sections is found by choosing $M_{1}, M_{2}, \mu, \tan \beta$ and the first generation slepton masses as in eq. (3.6), see table 12. 


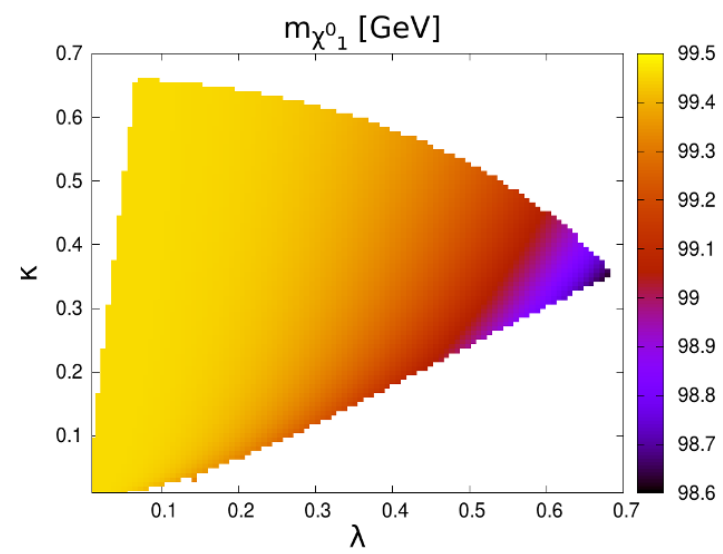

Figure 10. The LG scenario: the mass $m_{\tilde{\chi}_{1}^{0}}[\mathrm{GeV}]$.

\begin{tabular}{|c|c|c|c|c|c|}
\hline$m_{\tilde{\chi}_{1}^{0}}$ & $m_{\tilde{\chi}_{2}^{0}}$ & $m_{\tilde{\chi}_{3}^{0}}$ & $m_{\tilde{\chi}_{4}^{0}}$ & $m_{\tilde{\chi}_{1}^{ \pm}}$ & $m_{\tilde{\chi}_{2}^{ \pm}}$ \\
\hline $99.5 \mathrm{GeV}$ & $237.0 \mathrm{GeV}$ & $510.1 \mathrm{GeV}$ & $518.7 \mathrm{GeV}$ & $99.6 \mathrm{GeV}$ & $518.7 \mathrm{GeV}$ \\
\hline
\end{tabular}

\begin{tabular}{|c|c|c|}
\hline MSSM, $\sigma\left(e^{+} e^{-} \rightarrow \tilde{\chi}_{1}^{0} \tilde{\chi}_{2}^{0}\right)$ & $\sqrt{s}=350 \mathrm{GeV}$ & $\sqrt{s}=500 \mathrm{GeV}$ \\
\hline$P=(-0.9,0.55)$ & $7.3 \mathrm{fb}$ & $113.4 \mathrm{fb}$ \\
\hline$P=(0.9,-0.55)$ & $0.1 \mathrm{fb}$ & $1.8 \mathrm{fb}$ \\
\hline
\end{tabular}

\begin{tabular}{|c|c|c|}
\hline MSSM, $\sigma\left(e^{+} e^{-} \rightarrow \tilde{\chi}_{1}^{+} \tilde{\chi}_{1}^{-}\right)$ & $\sqrt{s}=350 \mathrm{GeV}$ & $\sqrt{s}=500 \mathrm{GeV}$ \\
\hline$P=(-0.9,0.55)$ & $2692.1 \mathrm{fb}$ & $1252.6 \mathrm{fb}$ \\
\hline$P=(0.9,-0.55)$ & $44.5 \mathrm{fb}$ & $19.4 \mathrm{fb}$ \\
\hline
\end{tabular}

Table 12. The reference light gaugino MSSM scenario: neutralino and chargino masses [GeV] and production cross sections $\sigma\left(e^{+} e^{-} \rightarrow \tilde{\chi}_{1}^{0} \tilde{\chi}_{2}^{0}\right), \sigma\left(e^{+} e^{-} \rightarrow \tilde{\chi}_{1}^{+} \tilde{\chi}_{1}^{-}\right)[\mathrm{fb}]$.

\begin{tabular}{|c|c|c|c|c|c|c|}
\hline$m_{\tilde{\chi}_{1}^{0}}$ & $m_{\tilde{\chi}_{2}^{0}}$ & $m_{\tilde{\chi}_{3}^{0}}$ & $m_{\tilde{\chi}_{4}^{0}}$ & $m_{\tilde{\chi}_{5}^{0}}$ & $m_{\tilde{\chi}_{1}^{ \pm}}$ & $m_{\tilde{\chi}_{2}^{ \pm}}$ \\
\hline $99.4 \mathrm{GeV}$ & $237.0 \mathrm{GeV}$ & $510.4 \mathrm{GeV}$ & $518.3 \mathrm{GeV}$ & $1768.2 \mathrm{GeV}$ & $99.5 \mathrm{GeV}$ & $518.7 \mathrm{GeV}$ \\
\hline
\end{tabular}

Table 13. Neutralino and chargino masses in the light gaugino scenario for the reference point LG1 with $(\lambda, \kappa)=(0.2,0.35)$.

In the LG scenario $m_{\tilde{\chi}_{1}^{0}}$ is very close to the reference MSSM value $99.5 \mathrm{GeV}$ and it varies very mildly in the $(\lambda, \kappa)$-plane as the singlino component in $\tilde{\chi}_{1}^{0}$ is approximately zero, see figure 10. A similar reasoning applies to the production cross section $\sigma\left(e^{+} e^{-} \rightarrow \tilde{\chi}_{1}^{0} \tilde{\chi}_{2}^{0}\right)$, while the chargino production, $\sigma\left(e^{+} e^{-} \rightarrow \tilde{\chi}_{1}^{+} \tilde{\chi}_{1}^{-}\right)$, is exactly identical at the tree-level as explained in section 3.2 .

We only use cross sections larger than $1 \mathrm{fb}$ for $\chi^{2}$-fit to the MSSM. Figure 11(a) shows that our fit alone is not able to distinguish in this case between the two models, as basically every point in the allowed region is compatible with the MSSM. 


\begin{tabular}{|c|c|c|}
\hline$\sigma\left(e^{+} e^{-} \rightarrow \tilde{\chi}_{1}^{0} \tilde{\chi}_{2}^{0}\right)$ & $\sqrt{s}=350 \mathrm{GeV}$ & $\sqrt{s}=500 \mathrm{GeV}$ \\
\hline$P=(-0.9,0.55)$ & $7.3 \mathrm{fb}$ & $113.5 \mathrm{fb}$ \\
\hline$P=(0.9,-0.55)$ & $0.1 \mathrm{fb}$ & $1.8 \mathrm{fb}$ \\
\hline
\end{tabular}

Table 14. Neutralino production cross sections in the light gaugino scenario for the reference point LG1 with $(\lambda, \kappa)=(0.2,0.35)$.

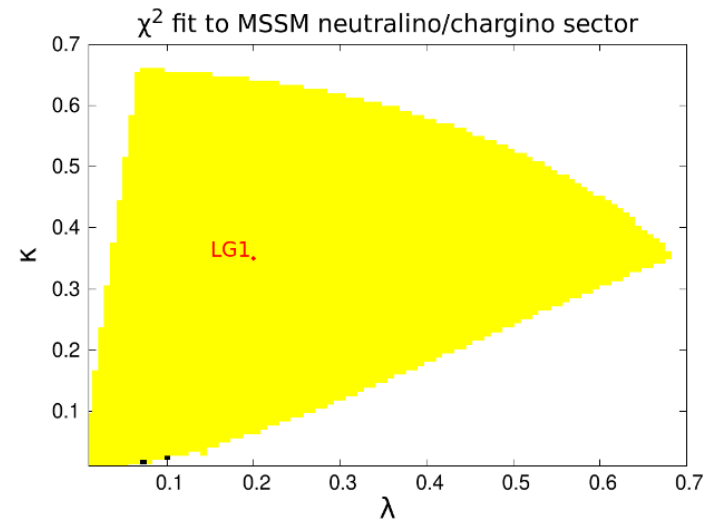

(a)

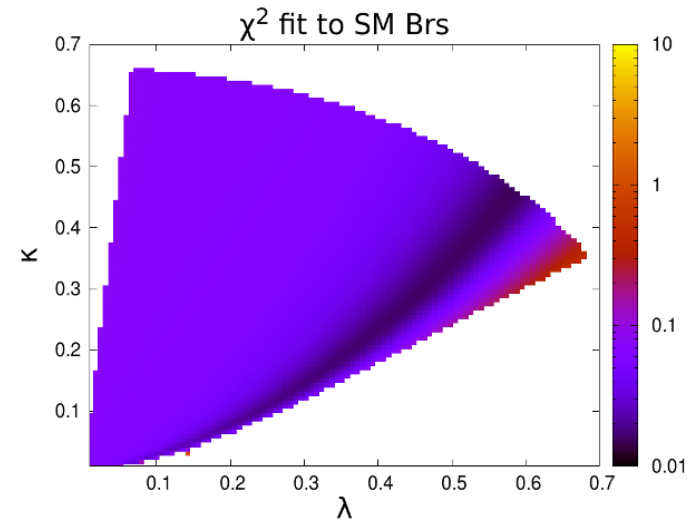

(b)

Figure 11. LG scenario: (a) fit to the MSSM. Yellow areas are compatible with the MSSM at $95 \%$ C.L., while black ones are excluded by the collider observables. The point LG1 $(\lambda, \kappa)=(0.2$, 0.35) is displayed. (b) $\chi^{2}$-fit to the SM of the reduced Higgs boson couplings to $g, \gamma, W, Z, b, c, \tau$.

As example we analyse the point LG1 with $(\lambda, \kappa)=(0.2,0.35)$ and the remaining parameters given by eqs. (3.6) and (3.7) that features the masses and cross sections listed in tables 13 and 14. For $P=(0.9,-0.55)$ the cross section $\sigma\left(e^{+} e^{-} \rightarrow \tilde{\chi}_{1}^{0} \tilde{\chi}_{2}^{0}\right)$ at $\sqrt{s}=350 \mathrm{GeV}$ is below $1 \mathrm{fb}$ and the process $e^{+} e^{-} \rightarrow \tilde{\chi}_{1}^{0} \tilde{\chi}_{3}^{0}$ is kinematically not allowed for both at 350 and $500 \mathrm{GeV}$. The remaining observables lead to a fit that is compatible with the MSSM giving $\chi^{2}=0.07$ :

$$
\begin{array}{rlrl}
M_{1} & =239.9 \pm 0.9 \mathrm{GeV}, & M_{2} & =104.4 \pm 0.8 \mathrm{GeV}, \\
\mu & =504.7 \pm 47.6 \mathrm{GeV}, & \tan \beta & =11.4 \pm 2.8, \\
m_{\tilde{\nu}_{e}} & =292.8 \pm 3.9 \mathrm{GeV} . &
\end{array}
$$

These values are remarkably close to the 'true' input parameters given by eqs. (3.6) and (3.7). A naïve fit of the SM-like Higgs reduced couplings does not provide information useful for model distinction, see figure 11(b), as they are always compatible with the SM, unlike in the LH scenario.

This behaviour can be understood by analysing the mixing within the neutralino sector. In the NMSSM, the singlino does not mix directly with gauginos but only indirectly via higgsino states, see eq. (A.7) and appendix A.1. If, like in the LG scenario, $\mu_{\mathrm{eff}} \gg M_{1}, M_{2}$, the mixing remains small even for a relatively light singlino. Therefore the properties of the light chargino and neutralino states, including masses and cross sections, remain 
very similar throughout the $(\lambda, \kappa)$-plane and cannot be distinguished from the MSSM case. In contrast to that, in the light singlino scenario from section $3.1, M_{1}=365 \mathrm{GeV}$ and $\mu_{\mathrm{eff}}=484 \mathrm{GeV}$ are of the similar size resulting in significant mixing: $\tilde{\chi}_{2}^{0} \simeq 22 \% \tilde{B}+73 \% \tilde{S}$ and $\tilde{\chi}_{3}^{0} \simeq 72 \% \tilde{B}+25 \% \tilde{S}$. Since in the LS case the singlino component makes up a significant part of the light neutralinos, the modification of the couplings allows the clear discrimination from the MSSM.

\section{Conclusions and outlook}

It will be very important to develop methods how to discriminate between the NMSSM and the MSSM at future experiments, as the two models may reproduce experimentally very similar light Higgs sectors as well as lower supersymmetric spectra. In this paper we have outlined a model distinction strategy that focuses on the neutralino and chargino sector and we have applied it to a series of NMSSM scenarios with different singlino, gaugino and higgsino properties. The idea is to assume that the lightest neutralino and chargino masses as well as their polarised pair production cross sections are measurable at a linear collider and to reconstruct the corresponding MSSM parameters, $M_{1}, M_{2}, \mu, \tan \beta$, via a $\chi^{2}$-fit. In case such a fit clearly excludes the MSSM hypothesis it would strongly point towards an extended model, preferably the NMSSM. Integrating the analysis with further information from the Higgs sector or from heavier neutralino resonances could confirm such a new model hypothesis. Throughout our study we have assumed to operate at the ILC with two different energy stages, namely at $\sqrt{s}=350$ and $500 \mathrm{GeV}$, using electron and positron beam polarisation with $P=( \pm 0.9, \mp 0.55)$.

We have introduced three classes of scenarios with different phenomenological aspects concerning the model distinction: a light singlino, a light higgsino and a light gaugino scenario. We have first analysed an NMSSM scenario with singlino components in the $\tilde{\chi}_{2}^{0}$ and a wino-like LSP $\tilde{\chi}_{1}^{0}$, i.e. with an inverted hierarchy of the gaugino mass parameters. Such a NMSSM scenario does not result in set of observables consistent with the MSSM. Accessing the mixing character of the heavier neutralino $\tilde{\chi}_{3}^{0}$ would confirm the situation and point to a model with an extended neutralino sector with respect to the MSSM.

In the class with light higgsinos, one usually has the hierarchy $\mu_{\text {eff }}<M_{1}<M_{2}$. In the corresponding NMSSM parameter space, a large part of the $(\lambda, \kappa)$-plane features the heavy and decoupled singlino while the $\tilde{\chi}_{1}^{0}$ is higgsino-like. Such a model is indistinguishable from the MSSM. However, if a sufficient singlino admixture is present in the light neutralinos, the neutralino sector changes appreciably, allowing for a discrimination between the MSSM and the NMSSM. In some region of the parameter space additional pseudoscalar Higgs $a_{1}$ could also be observed. Precise measurement of the SM-like Higgs couplings would be beneficial for a confirmation of these conclusions.

As a third class we have chosen light gaugino scenarios again with an inverted hierarchy $M_{2}<M_{1}<\mu_{\text {eff }}$ but with $\mu_{\text {eff }}-M_{1} \sim \mathcal{O}(250) \mathrm{GeV}$. In this way the singlino does not significantly mix with gauginos in the lightest neutralino states. In the light of our experimental assumptions, the low mass spectrum and production cross sections are not distinguishable 
from the MSSM ones all over the allowed $(\lambda, \kappa)$-plane. In this case analysing the SM-like Higgs couplings also does not provide further information.

Our studies show that the neutralino and chargino sector can provide the crucial information for the model distinction between the MSSM and the NMSSM. Such a discrimination depends on the gaugino mass hierarchies and the actual singlino admixture in the light neutralino states. Precise measurements and a model-independent analysis for the determination of the fundamental SUSY parameters are essential.

\section{Acknowledgments}

The authors are thankful to M. Berggren, F. Domingo, P. Gunnellini, J. List, O. Stål, M. Tonini, M. de Vries, G. Weiglein and L. Zeune for useful discussions. S. P. has been supported by DFG through the grant SFB 676 "Particles, Strings, and the Early Universe". This work has been partially supported by the MICINN, Spain, under contract FPA2010-17747; Consolider-Ingenio CPAN CSD2007-00042. We thank also the Comunidad de Madrid through Proyecto HEPHACOS S2009/ESP-1473 and the European Commission under contract PITN-GA-2009-237920.

\section{A Chargino, neutralino and Higgs sector}

\section{A.1 Chargino and neutralino mass matrices}

The tree-level chargino sector is identical for the MSSM and NMSSM. In the $\left(\tilde{W}^{ \pm}, \tilde{H}^{ \pm}\right)$ basis, the chargino mass matrix reads

$$
\mathcal{M}_{C}=\left(\begin{array}{cc}
M_{2} & \sqrt{2} m_{Z} \cos \theta_{W} \cos \beta \\
\sqrt{2} m_{Z} \cos \theta_{W} \sin \beta & \mu
\end{array}\right),
$$

in the convention according to which $\tilde{\chi}^{-}$is taken as the particle and $\tilde{\chi}^{+}$as its antiparticle (i.e. different convention as in e.g. [39]). $M_{2}$ is chosen real and positive. The charginos, eigenstates of $\mathcal{M}_{C}$, can be written as [14]

$$
\left(\begin{array}{c}
\tilde{\chi}_{1}^{-} \\
\tilde{\chi}_{2}^{-}
\end{array}\right)_{L, R}=U_{L, R}\left(\begin{array}{c}
\tilde{W}^{-} \\
\tilde{H}^{-}
\end{array}\right)_{L, R}=\left(\begin{array}{cc}
\cos \Phi_{L, R} & \sin \Phi_{L, R} \\
-\sin \Phi_{L, R} & \cos \Phi_{L, R}
\end{array}\right)\left(\begin{array}{c}
\tilde{W}^{-} \\
\tilde{H}^{-}
\end{array}\right)_{L, R},
$$

such that

$$
\begin{aligned}
m_{\tilde{\chi}_{1,2}^{ \pm}}^{2} & =\frac{1}{2}\left(M_{2}^{2}+\mu^{2}+2 m_{W}^{2} \mp \Delta_{C}\right), \\
\cos 2 \Phi_{L, R} & =-\left(M_{2}^{2}-\mu^{2} \mp 2 m_{W}^{2} \cos 2 \beta\right) / \Delta_{C},
\end{aligned}
$$

where $\Delta_{C}=\left[\left(M_{2}^{2}-\mu^{2}\right)^{2}+4 m_{W}^{4} \cos ^{2} 2 \beta+4 m_{W}^{2}\left(M_{2}^{2}+\mu^{2}\right)+8 m_{W}^{2} M_{2} \mu \sin 2 \beta\right]^{1 / 2}$.

The tree-level MSSM neutralino mass matrix in the $\left(\tilde{B}, \tilde{W}^{0}, \tilde{H}_{d}, \tilde{H}_{u}\right)$ basis,

$$
\mathcal{M}_{\mathrm{MSSM}}=\left(\begin{array}{cccc}
M_{1} & 0 & -\cos \beta \sin \theta_{W} m_{Z} & \sin \beta \sin \theta_{W} m_{Z} \\
0 & M_{2} & \cos \beta \cos \theta_{W} m_{Z} & -\sin \beta \cos \theta_{W} m_{Z} \\
-\cos \beta \sin \theta_{W} m_{Z} & \cos \beta \cos \theta_{W} m_{Z} & 0 & -\mu \\
\sin \beta \sin \theta_{W} m_{Z} & -\sin \beta \cos \theta_{W} m_{Z} & -\mu & 0
\end{array}\right) \text {, }
$$


can be diagonalised by a unitary matrix $N$, obtaining the neutralino eigenvectors and their masses:

$$
N^{*} \mathcal{M}_{\mathrm{MSSM}} N^{\dagger}=\operatorname{diag}\left\{m_{\tilde{\chi}_{1}^{0}}, \ldots, m_{\tilde{\chi}_{4}^{0}}\right\} .
$$

$\mathcal{M}_{\text {MSSM }}$ is equivalent to the upper left block of the the tree-level ( $\mathbb{Z}_{3}$-invariant) NMSSM neutralino mass matrix, in the basis $\left(\tilde{\gamma}, \tilde{Z}, \tilde{H}_{d}, \tilde{H}_{u}, \tilde{S}\right)[6]$ :

$$
\mathcal{M}_{\mathrm{NMSSM}}=\left(\begin{array}{cc} 
& 0 \\
\mathcal{M}_{\mathrm{MSSM}} & 0 \\
& -\lambda v \sin \beta \\
& -\lambda v \cos \beta \\
\hline 00-\lambda v \sin \beta-\lambda v \cos \beta & -2 \kappa \mu_{\mathrm{eff}} / \lambda
\end{array}\right),
$$

with the only difference that now $\mu$ is substituted by $\mu_{\text {eff }}=\lambda s$, where $s$ the vev of the singlet, and where $v_{u}^{2}+v_{d}^{2}=v^{2}=2 m_{Z}^{2} /\left(g_{1}^{2}+g_{2}^{2}\right) \approx(174 \mathrm{GeV})^{2}$. The NMSSM neutralino sector depends on two more singlet/singlino parameters with respect to the MSSM: $\lambda, \kappa$, while $\mu$ dependence is substituted by the dependence on the singlet vev $s$.

\section{A.2 $\mathbb{Z}_{3}$-NMSSM Higgs sector}

According to ref. [6], for the $\mathbb{Z}_{3}$-invariant NMSSM, the part of the superpotential describing Higgs-Singlet (self) interactions is given by:

$$
W_{\text {Higgs-singlet }}=\lambda \hat{S} \hat{H}_{u} \cdot \hat{H}_{d}+\frac{\kappa}{3} \hat{S}^{3},
$$

while the Yukawa couplings are described by

$$
W_{\text {Yukawa }}=h_{u} \hat{Q} \cdot \hat{H}_{u} \hat{U}_{R}^{c}+h_{d} \hat{H}_{d} \cdot \hat{Q} \hat{D}_{R}^{c}+h_{e} \hat{H}_{d} \cdot \hat{L} \hat{E}_{R}^{c} .
$$

The Higgs soft SUSY breaking lagrangian reads:

$$
\begin{aligned}
-\mathcal{L}_{\text {Higgs-Singlet soft }}= & h_{u} A_{u} Q \cdot H_{u} U_{R}^{c}-h_{d} A_{d} Q \cdot D_{R}^{c}-h_{e} A_{e} L \cdot H_{d} E_{R}^{c} \\
& +\lambda A_{\lambda} H_{u} \cdot H_{d} S+\frac{\kappa}{3} A_{\kappa} S^{3}+\text { h.c. }
\end{aligned}
$$

From eqs. (A.8) and (A.10) one obtains the Higgs scalar potential

$$
\begin{aligned}
V_{\text {Higgs }}= & \left|\lambda\left(H_{u}^{+} H_{d}^{-}-H_{u}^{0} H_{d}^{0}\right)+\kappa S\right|^{2} \\
& +\left(m_{H_{u}}^{2}+|\mu+\lambda S|^{2}\right)\left(\left|H_{u}^{0}\right|^{2}+\left|H_{u}^{+}\right|^{2}\right)+\left(m_{H_{d}}^{2}+|\mu+\lambda S|^{2}\right)\left(\left|H_{d}^{0}\right|^{2}+\left|H_{d}^{-}\right|^{2}\right) \\
& +\frac{g_{1}^{2}+g_{2}^{2}}{8}\left(\left|H_{u}^{0}\right|^{2}+\left|H_{u}^{+}\right|^{2}-\left|H_{d}^{0}\right|^{2}-\left|H_{d}^{-}\right|^{2}\right)+\frac{g_{2}^{2}}{2}\left|H_{u}^{+} H_{d}^{0 *}+H_{u}^{0} H_{d}^{-*}\right|^{2} \\
& +m_{S}^{2}|S|^{2}+\left(\lambda A_{\lambda}\left(H_{u}^{+} H_{d}^{-}-H_{u}^{0} H_{d}^{0}\right) S+\frac{k}{3} A_{\kappa} S^{3}+\text { h.c. }\right)
\end{aligned}
$$


from which one derives the Higgs mass eigenstates. Conventionally, we take

$$
H_{u}^{0}=v_{u}+\frac{H_{u R}+i H_{u I}}{\sqrt{2}}, \quad H_{d}^{0}=v_{d}+\frac{H_{d R}+i H_{d I}}{\sqrt{2}}, \quad S=s+\frac{S_{R}+i S_{I}}{\sqrt{2}} .
$$

We define $\mu_{\mathrm{eff}}=\lambda s$, so the CP-even Higgs mass matrix is given by

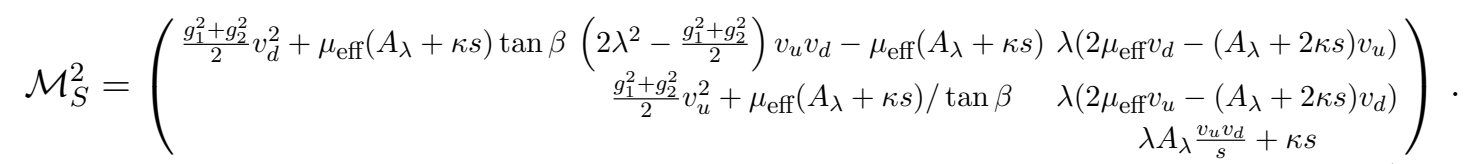

The CP-odd Higgs mass matrix reads:

$$
\mathcal{M}_{P}^{2}=\left(\begin{array}{cc}
2 \mu_{\mathrm{eff}}\left(A_{\lambda}+\kappa s\right) / \sin 2 \beta & \lambda\left(A_{\lambda}-2 \kappa s\right) v \\
& \lambda\left(A_{\lambda}+4 \kappa s\right) \frac{v_{u} v_{d}}{s}-3 \kappa A_{\kappa} s
\end{array}\right) .
$$

Finally, the NMSSM charged Higgs states $H^{ \pm}$have the mass:

$$
m_{H^{ \pm}}^{2}=\frac{2 \mu_{\mathrm{eff}}\left(A_{\lambda}+\kappa s\right)}{\sin 2 \beta}+v^{2}\left(\frac{g_{2}^{2}}{2}-\lambda^{2}\right) .
$$

\section{B Scenarios}

\section{B.1 Light singlino scenario}

Parameters at the EWSB scale $(2 \mathrm{TeV})$ are listed in table 15 .

\begin{tabular}{|c|c|c|c|c|c|c|}
\hline$M_{1}$ & $M_{2}$ & $M_{3}$ & $\tan \beta$ & $\mu_{\mathrm{eff}}=\lambda s$ & $A_{\lambda}$ & $A_{\kappa}$ \\
\hline $365 \mathrm{GeV}$ & $111 \mathrm{GeV}$ & $2000 \mathrm{GeV}$ & 9.5 & $484 \mathrm{GeV}$ & $4200 \mathrm{GeV}$ & $-120 \mathrm{GeV}$ \\
\hline
\end{tabular}

\begin{tabular}{|c|c|c|c|c|c|c|}
\hline$M_{Q_{1,2}}, M_{u_{1,2}}, M_{d_{1,2}}$ & $M_{Q_{3}}$ & $M_{u_{3}}$ & $M_{d_{3}}$ & $M_{l}, M_{e}$ & $A_{u_{3}}$ & $A_{d_{3}}, A_{e_{3}}$ \\
\hline $2000 \mathrm{GeV}$ & $1500 \mathrm{GeV}$ & $1000 \mathrm{GeV}$ & $800 \mathrm{GeV}$ & $300 \mathrm{GeV}$ & $2750 \mathrm{GeV}$ & $2000 \mathrm{GeV}$ \\
\hline
\end{tabular}

Table 15. Parameters of LS scenario, at the EWSB scale.

\section{B.2 Light higgsino scenario}

Parameters at the EWSB scale $(2 \mathrm{TeV})$ are listed in table 16 .

\begin{tabular}{|c|c|c|c|c|c|c|}
\hline$M_{1}$ & $M_{2}$ & $M_{3}$ & $\tan \beta$ & $\mu_{\mathrm{eff}}=\lambda s$ & $A_{\lambda}$ & $A_{\kappa}$ \\
\hline $450 \mathrm{GeV}$ & $1600 \mathrm{GeV}$ & $2000 \mathrm{GeV}$ & 27 & $120 \mathrm{GeV}$ & $3000 \mathrm{GeV}$ & $-30 \mathrm{GeV}$ \\
\hline$M_{Q_{1,2}}, M_{u_{1,2}}, M_{d_{1,2}}$ & $M_{Q_{3}}, M_{u_{3}}, M_{d_{3}}$ & $M_{l}, M_{e}$ & $A_{u_{3}}$ & $A_{d_{3}}, A_{e_{3}}$ \\
\hline \multicolumn{2}{|c|}{$1500 \mathrm{GeV}$} & $300 \mathrm{GeV}$ & $3300 \mathrm{GeV}$ & $200 \mathrm{GeV}$ \\
\hline
\end{tabular}

Table 16. Parameters of LH scenario, at the EWSB scale. 


\section{B.3 Light gaugino scenario}

Parameters at the EWSB scale $(2 \mathrm{TeV})$ are listed in table 17.

\begin{tabular}{|c|c|c|c|c|c|c|}
\hline$M_{1}$ & $M_{2}$ & $M_{3}$ & $\tan \beta$ & $\mu_{\text {eff }}=\lambda s$ & $A_{\lambda}$ & $A_{\kappa}$ \\
\hline $240 \mathrm{GeV}$ & $105 \mathrm{GeV}$ & $2000 \mathrm{GeV}$ & 9.2 & $505 \mathrm{GeV}$ & $3700 \mathrm{GeV}$ & $-40 \mathrm{GeV}$ \\
\hline
\end{tabular}

\begin{tabular}{|c|c|c|c|c|c|c|c|}
\hline$M_{Q_{1,2}}, M_{u_{1,2}}, M_{d_{1,2}}$ & $M_{Q_{3}}$ & $M_{u_{3}}, M_{d_{3}}$ & $M_{l_{1,2}}, M_{e_{1,2}}$ & $M_{l_{3}}, M_{e_{3}}$ & $A_{u_{3}}$ & $A_{d_{3}}$ & $A_{e_{3}}$ \\
\hline $2000 \mathrm{GeV}$ & $1800 \mathrm{GeV}$ & $1500 \mathrm{GeV}$ & $300 \mathrm{GeV}$ & $500 \mathrm{GeV}$ & $3700 \mathrm{GeV}$ & $2500 \mathrm{GeV}$ & $1500 \mathrm{GeV}$ \\
\hline
\end{tabular}

Table 17. Parameters of LG scenario, at the EWSB scale.

Open Access. This article is distributed under the terms of the Creative Commons Attribution License (CC-BY 4.0), which permits any use, distribution and reproduction in any medium, provided the original author(s) and source are credited.

\section{References}

[1] ATLAS collaboration, Observation of a new particle in the search for the standard model Higgs boson with the ATLAS detector at the LHC, Phys. Lett. B 716 (2012) 1 [arXiv: 1207.7214] [INSPIRE].

[2] CMS collaboration, Observation of a new boson at a mass of $125 \mathrm{GeV}$ with the CMS experiment at the LHC, Phys. Lett. B 716 (2012) 30 [arXiv:1207.7235] [INSPIRE].

[3] CMS collaboration, Measurement of the properties of a Higgs boson in the four-lepton final state, Phys. Rev. D 89 (2014) 092007 [arXiv: 1312.5353] [INSPIRE].

[4] ATLAS collaboration, Measurement of the Higgs boson mass from the $H \rightarrow \gamma \gamma$ and $H \rightarrow Z Z^{*} \rightarrow 4 \ell$ channels with the ATLAS detector using $25 \mathrm{fb}^{-1}$ of pp collision data, arXiv: 1406.3827 [INSPIRE].

[5] S.P. Martin, A supersymmetry primer, Adv. Ser. Direct. High Energy Phys. 21 (2010) 1 [hep-ph/9709356] [INSPIRE].

[6] U. Ellwanger, C. Hugonie and A.M. Teixeira, The next-to-minimal supersymmetric standard model, Phys. Rept. 496 (2010) 1 [arXiv:0910.1785] [inSPIRE].

[7] ATLAS collaboration, A general search for new phenomena with the ATLAS detector in pp collisions at $\sqrt{s}=8 \mathrm{TeV}$, ATLAS-CONF-2014-006 (2014).

[8] CMS collaboration, Phenomenological MSSM interpretation of the CMS 7 and 8 TeV results, CMS-PAS-SUS-13-020 (2013).

[9] R.S. Gupta, M. Montull and F. Riva, SUSY faces its Higgs couplings, JHEP 04 (2013) 132 [arXiv: 1212.5240] [INSPIRE].

[10] D.M. Asner et al., ILC Higgs white paper, arXiv:1310.0763 [INSPIRE].

[11] S.Y. Choi et al., Reconstructing the chargino system at $e^{+} e^{-}$linear colliders, Eur. Phys. J. C 14 (2000) 535 [hep-ph/0002033] [INSPIRE].

[12] S.Y. Choi, J. Kalinowski, G.A. Moortgat-Pick and P.M. Zerwas, Analysis of the neutralino system in supersymmetric theories, Eur. Phys. J. C 22 (2001) 563 [Addendum ibid. C 23 (2002) 769-772] [hep-ph/0108117] [INSPIRE]. 
[13] S.Y. Choi, J. Kalinowski, G.A. Moortgat-Pick and P.M. Zerwas, Analysis of the neutralino system in supersymmetric theories: addendum, hep-ph/0202039 [INSPIRE].

[14] K. Desch, J. Kalinowski, G.A. Moortgat-Pick, M.M. Nojiri and G. Polesello, SUSY parameter determination in combined analyses at LHC/LC, JHEP 02 (2004) 035 [hep-ph/0312069] [INSPIRE].

[15] K. Desch, J. Kalinowski, G. Moortgat-Pick, K. Rolbiecki and W.J. Stirling, Combined LHC/ILC analysis of a SUSY scenario with heavy sfermions, JHEP 12 (2006) 007 [hep-ph/0607104] [INSPIRE].

[16] G.A. Moortgat-Pick, S. Hesselbach, F. Franke and H. Fraas, Distinguishing between MSSM and NMSSM by combined LHC and ilc analyses, JHEP 06 (2005) 048 [hep-ph/0502036] [INSPIRE].

[17] S. Porto, G.A. Moortgat-Pick and K. Rolbiecki, Towards discrimination of MSSM and NMSSM scenarios at colliders, arXiv:1404.1053 [INSPIRE].

[18] J.-J. Cao, Z.-X. Heng, J.M. Yang, Y.-M. Zhang and J.-Y. Zhu, A SM-like Higgs near 125 GeV in low energy SUSY: a comparative study for MSSM and NMSSM, JHEP 03 (2012) 086 [arXiv: 1202.5821] [INSPIRE].

[19] J. Cao, Z. Heng, J.M. Yang and J. Zhu, Status of low energy SUSY models confronted with the LHC 125 GeV Higgs data, JHEP 10 (2012) 079 [arXiv:1207.3698] [INSPIRE].

[20] R. Benbrik et al., Confronting the MSSM and the NMSSM with the discovery of a signal in the two photon channel at the LHC, Eur. Phys. J. C 72 (2012) 2171 [arXiv:1207.1096] [INSPIRE].

[21] C. Beskidt, W. de Boer and D.I. Kazakov, A comparison of the Higgs sectors of the CMSSM and NMSSM for a 126 GeV Higgs boson, Phys. Lett. B 726 (2013) 758 [arXiv:1308.1333] [INSPIRE].

[22] P.N. Pandita and M. Patra, Invisible decays of low mass Higgs bosons in supersymmetric models, Phys. Rev. D 89 (2014) 115010 [arXiv:1405.7163] [inSPIRE].

[23] U. Ellwanger, J.F. Gunion, C. Hugonie and S. Moretti, Towards a no lose theorem for NMSSM Higgs discovery at the LHC, hep-ph/0305109 [INSPIRE].

[24] A. Bharucha, J. Kalinowski, G. Moortgat-Pick, K. Rolbiecki and G. Weiglein, One-loop effects on MSSM parameter determination via chargino production at the LC, Eur. Phys. J. C 73 (2013) 2446 [arXiv:1211.3745] [INSPIRE].

[25] U. Ellwanger, J.F. Gunion and C. Hugonie, NMHDECAY: a Fortran code for the Higgs masses, couplings and decay widths in the NMSSM, JHEP 02 (2005) 066 [hep-ph/0406215] [INSPIRE].

[26] U. Ellwanger and C. Hugonie, NMHDECAY 2.0: an updated program for sparticle masses, Higgs masses, couplings and decay widths in the NMSSM, Comput. Phys. Commun. 175 (2006) 290 [hep-ph/0508022] [INSPIRE].

[27] G. Bélanger, F. Boudjema, C. Hugonie, A. Pukhov and A. Semenov, Relic density of dark matter in the NMSSM, JCAP 09 (2005) 001 [hep-ph/0505142] [INSPIRE].

[28] D. Das, U. Ellwanger and A.M. Teixeira, NMSDECAY: a Fortran Code for supersymmetric particle decays in the next-to-minimal supersymmetric standard model, Comput. Phys. Commun. 183 (2012) 774 [arXiv:1106.5633] [INSPIRE]. 
[29] M. Muhlleitner, A. Djouadi and Y. Mambrini, SDECAY: a Fortran code for the decays of the supersymmetric particles in the MSSM, Comput. Phys. Commun. 168 (2005) 46 [hep-ph/0311167] [INSPIRE].

[30] G. Bélanger, F. Boudjema, A. Pukhov and A. Semenov, MicrOMEGAs_3: a program for calculating dark matter observables, Comput. Phys. Commun. 185 (2014) 960 [arXiv: 1305.0237] [INSPIRE].

[31] LUX collaboration, D.S. Akerib et al., First results from the LUX dark matter experiment at the Sanford Underground Research Facility, Phys. Rev. Lett. 112 (2014) 091303 [arXiv: 1310.8214] [INSPIRE].

[32] Planck collaboration, P.A.R. Ade et al., Planck 2013 results. XVI. Cosmological parameters, Astron. Astrophys. (2014) [arXiv:1303.5076] [INSPIRE].

[33] P. Bechtle et al., HiggsBounds-4: improved tests of extended Higgs sectors against exclusion bounds from LEP, the Tevatron and the LHC, Eur. Phys. J. C 74 (2014) 2693 [arXiv:1311.0055] [INSPIRE].

[34] P. Bechtle, S. Heinemeyer, O. Stål, T. Stefaniak and G. Weiglein, HiggsSignals: confronting arbitrary Higgs sectors with measurements at the Tevatron and the LHC, Eur. Phys. J. C 74 (2014) 2711 [arXiv: 1305.1933] [INSPIRE].

[35] ECFA/DESY LC Physics Working Group collaboration, J.A. Aguilar-Saavedra et al., TESLA: the superconducting electron positron linear collider with an integrated $x$-ray laser laboratory. Technical design report. Part 3. Physics at an $e^{+} e^{-}$linear collider, hep-ph/0106315 [INSPIRE].

[36] H. Baer, T. Barklow, K. Fujii, Y. Gao, A. Hoang et al., The International Linear Collider technical design report - Volume 2: physics, arXiv:1306.6352 [INSPIRE].

[37] F. James and M. Roos, Minuit - A system for function minimization and analysis of the parameter errors and correlations, Comput. Phys. Commun. 10 (1975) 343.

[38] M. Berggren et al., Tackling light higgsinos at the ILC, Eur. Phys. J. C 73 (2013) 2660 [arXiv: 1307.3566] [INSPIRE].

[39] H.E. Haber and G.L. Kane, The search for supersymmetry: probing physics beyond the standard model, Phys. Rept. 117 (1985) 75 [INSPIRE]. 